\title{
INVERTING A CYLINDER THROUGH ISOMETRIC IMMERSIONS AND ISOMETRIC EMBEDDINGS
}

BY

\author{
B. HALPERN AND C. WEAVER
}

\begin{abstract}
It is shown that a right circular cylinder can be turned inside out through immersions which preserve its flat Riemannian metric if and only if its diameter is greater than its height. Such a cylinder can be turned inside out through embeddings which preserve its flat Riemannian metric provided its diameter is greater than $(\pi+2) / \pi$ times its height. A flat Möbius strip has an immersion into Euclidean three dimensional space which preserves its Riemannian metric if and only if its length is greater than $\pi / 2$ times its height.
\end{abstract}

1. Introduction. A smooth map from one smooth Riemannian manifold to another is said to be isometric if it preserves the Riemannian metric. Two isometric maps $f, g: M \rightarrow N$, are isometrically homotopic (isotopic) provided there is a smooth homotopy $H_{t}(x)$ from $f$ to $g$ such that, for each $t, H_{t}$ : $M \rightarrow N$ is an isometric immersion (embedding). We consider the case where $M$ is a cylinder and $N$ is Euclidean 3-space, $\mathbf{R}^{3}$. Let $C_{h}$ be the cylinder formed by taking the product of the unit circle $S^{1} \subset \mathbf{R}^{2}$ with the closed interval $I_{h}=[-h, h] \subset \mathbf{R}, C_{h}=S^{1} \times I_{h} \subset \mathbf{R}^{2} \times \mathbf{R}^{1}=\mathbf{R}^{3}$. Let $i: C_{h} \rightarrow \mathbf{R}^{3}$ be the inclusion map and give $C_{h}$ the Riemannian metric induced by $i$ and the standard metric on $\mathbf{R}^{3}$. Let $r: C_{h} \rightarrow \mathbf{R}^{3}$ be the map which turns $C_{h}$ inside out, $r(x, y, z)=(x, y,-z)$. Paper models of $C_{h}$ strongly suggest that for $h$ large (such a $C_{h}$ resembles a soda straw) $i$ and $r$ are not isometrically isotopic, and for $h$ small (here $C_{h}$ resembles a long band of ribbon) $i$ and $r$ are isometrically isotopic. We prove here the following results. The maps $i$ and $r$ are isometrically homotopic iff $h<1$. If $i$ and $r$ are isometrically isotopic, then $h<1$; if $h<\pi /(\pi+2)$, then $i$ and $r$ are isometrically isotopic. The proof occupies $\S \S 2-10$.

For each isometry $f: C_{h} \rightarrow \mathbf{R}^{3}$ we define an element $G(f)$ which lies in $\mathbf{Z}$ for $h \geqslant 1$, and in $\mathbf{Z}_{2}$ for $h<1$, and which is invariant under isometric

Presented to the Society, August 25, 1970 under the title Bending and immersing strips and bands; received by the editors September 9, 1975.

AMS (MOS') subject classifications (1970). Primary 58D10; Secondary 57D40.

Key words and phrases. Isometric immersion, isometric embedding, homotopy, isotopy, Möbius band, flat cylinder. 
homotopy. For $f$ of the form $f=\gamma \times j: S^{1} \times I_{h} \rightarrow \mathbf{R}^{2} \times \mathbf{R}^{1}=\mathbf{R}^{3}$, where $\gamma$ : $S^{1} \rightarrow \mathbf{R}^{2}$ is a smooth closed curve and $j: I_{h} \rightarrow \mathbf{R}^{1}$ is the inclusion map, we have the formula

$$
G(f)= \begin{cases}\mathcal{W}(\gamma) & \text { for } h \geqslant 1, \\ \mathcal{W}(\gamma) \bmod 2 & \text { for } h<1,\end{cases}
$$

where $\mho(\gamma)$ is the tangent winding number of $\gamma$. For isometries of the form $f=\gamma \times j, G(f)$ completely classifies $f$ up to isometric homotopy. This we prove in $\S 11$.

Two closely related problems are those of isometrically immersing and isometrically embedding the Möbius band in $\mathbf{R}^{3}$. According to [11] it.has been an open question as to how long a strip of paper one needs in order to make a Möbius strip. There are two things which tend to require longer strips. One is the problem of twisting while preserving the flat Riemannian metric. The second is the problem of avoiding self-intersections so that one has an embedding. By considering isometric immersions one can concentrate on the first problem, which we have solved completely. We have partial results concerning the second problem. Let $M_{l}=([0, l] \times[0,1]) / \sim$ where $(0$, $y) \sim(1, l-y)$ for all $y, 0 \leqslant y \leqslant 1$. Give $M_{l}$ the obvious flat Riemannian metric. In $\S 12$ we show that $M_{l}$ isometrically immerses in $\mathbf{R}^{3}$ iff $l>\pi / 2$.

A homotopy through isometric immersions from $i$ to $r$ is called an inversion. Our example of an inversion for $h$ near 1 passes through a stage where $C_{h}$ is nearly folded up with many folds. In $\S 13$ we establish results which roughly say that any inversion for $h$ near 1 must also pass through a stage where $C_{h}$ is nearly folded up with many folds. These results are then applied in $\S 14$ to show that if we restrict ourselves to only isometric immersion with mean curvature bound by a fixed bound $B$, then there is an $\varepsilon>0$ such that there is an inversion only if $h<1-\varepsilon$.

In $\S 15$ we show that there exists an $\varepsilon>0$ such that if $M_{l}$ isometrically embeds in $\mathbf{R}^{3}$, then $l>\pi / 2+\varepsilon$, and if $l>\sqrt{3}$, then $M_{l}$ isometrically embeds in $\mathbf{R}^{3}$. This shows that there is an $l$ such that $M_{l}$ can be isometrically immersed in $\mathbf{R}^{3}$ but not isometrically embedded in $\mathbf{R}^{3}$. It is not known whether there is an $h$ for which $i$ is isometrically homotopic to $r$ but not isometrically isotopic to $r$.

Some previous studies of isometric homotopies have taken a different point of view. Several theorems have been established which say that certain manifolds in $\mathbf{R}^{n}$ are or are not rigid. A manifold $M \subset \mathbf{R}^{n}$ is said to be rigid provided the inclusion map $k: M \rightarrow \mathbf{R}^{n}$ is isometrically homotopic to only maps of the form $U \circ k$ where $U$ is an element of the special orthogonal group $S O(n)$. Also much work has been done on isometric embeddings and immersions. For a survey of results, see [4]. 
2. Rough description of $G(f)$. We give here a rough description of the invariant $G(f)$. A rigorous definition is given in $\$ 7$ after some necessary lemmas are established in $\S \S 3,4,5$ and 6.

The definition of $G(f)$ for $h<1$ is easy and we give a rigorous definition here. For each $p \in S^{1}$, let $N(p)$ be the unit normal to $f$ at $(p, 0)$, and let $T(p)$ be the unit tangent vector to the curve $f \mid S^{1} \times 0$. Set $G(f) \in \pi_{1}(S O(3)) \approx$ $\mathbf{Z}_{2}$ equal to the homotopy class of $\gamma: S^{1} \rightarrow S O(3)$ where $\gamma(p)=(T(p), N(p)$, $T(p) \times N(p))$. The following theorem is clear.

\subsection{Theorem. $G(f)$ is invariant under isometric homotopy.}

The following theorem is also immediate from the definition.

2.2. THEOREM. If $f$ is of the form

$$
f=\gamma \times j: S^{1} \times I_{h} \rightarrow \mathbf{R}^{2} \times \mathbf{R}^{1}=\mathbf{R}^{3} \text {, where } \gamma: S^{1} \rightarrow \mathbf{R}^{2}
$$

is a smooth closed curve and $j: I_{h} \rightarrow \mathbf{R}^{1}$ is the inclusion map, and $h<1$, then $G(f)=$ the tangent winding number of $\gamma$ reduced mod 2 .

Now suppose $f: C_{h} \rightarrow \mathbf{R}^{3}$ is an isometric immersion and $h>1$. We wish to define an integer $G(f)$ which is invariant under isometric homotopy. We start by considering a related map $g: \tilde{C}_{h} \rightarrow \mathbf{R}^{3}$ where $\tilde{C}_{h}$ is the strip of height $h$, $\tilde{C}_{h}=\mathbf{R} \times I_{h}, \pi: \tilde{C}_{h} \rightarrow C_{h}$ is the natural covering map, $\pi(s, t)=(\cos s, \sin s$, $t$ ), and $g=f \circ \pi$. Because $g$ is a flat surface it is ruled and there are plenty of straight line segments which go from one boundary of $\tilde{C}_{h}$ to the other and which are mapped into straight line segments in $\mathbf{R}^{3}$ by $g$ (see $\S 4$ ). In order to get a hold on these line segments, we define

$$
\begin{aligned}
& \tilde{V}(s)=\tilde{V}_{g}(s) \\
& =\{t \in \mathbf{R} \mid\|g(s+t, h)-g(s-t,-h)\|=\|(s+t, h)-(s-t,-h)\|\}
\end{aligned}
$$

and

$$
\tilde{W}(s)=\tilde{W}_{g}(s)=\left.d g\right|_{(s, 0)}\left(\tilde{V}_{g}(s)\right) \times h
$$

for $s \in \mathbf{R}$ where $\|\cdot\|$ is the Euclidean norm.

The condition $t \in \tilde{V}_{g}(s)$ means that $g$ maps the segment from $(s-t,-h)$ to $(s+t, h)$ rigidly. Hence $\tilde{W}(s)=\left\{g(s+t, h)-g(s, 0) \mid t \in \tilde{V}_{g}(s)\right\}$. Since $g=f \circ \pi$ it makes sense to define for each $p \in S^{1}$

$$
V(p)=V_{f}(p)=\tilde{V}_{g}(s) \text { and } W(p)=W_{f}(p)=\tilde{W}_{g}(s)
$$

where $s$ is any element of $\mathbf{R}^{1}$ such that $\pi(s, 0)=(p, 0)$.

The directed straight line segment from $g(s)-w$ to $g(s)+w$ where $w \in \tilde{W}_{g}(s)$ is called a generating line segment as it is easy to see that it lies entirely on the surface $g\left(\tilde{C}_{h}\right)=f\left(C_{h}\right)$, and in Proposition 4.1 we show that the totality of these segments "generates the surface". We will show in 
Proposition 5.1 that there do not exist two antiparallel generating line segments. Thus, if we "normalize" the surface $f\left(C_{h}\right)$ by rotating it so that a prescribed element of $\tilde{W}(0)$ points upward (parallel to $e_{3}=(0,0,1) \in \mathbf{R}^{3}$ ) we can assert that the downward pointing ray $R=\left\{t e_{3} \mid t \leqslant 0\right\}$ does not intersect any of the sets $W(p), R \cap W(p)=\varnothing$ for $p \in S^{1}$. Then we can define a related set-valued map $\bar{\varphi}: S^{1} \rightarrow 2^{Q}$ where $Q=\left\{(u, v) \in\left(\mathbf{R}^{3}-R\right) \times \mathbf{R}^{3} \mid u, v\right.$ are linearly independent $\}$ by setting $\bar{\varphi}(p)=(W(p), T(p))=\{(w, T(p)) \mid w \in$ $W(p)$ \}. Since $Q$ has $S^{1}$ as a deformation retract (see Lemma 6.1), $H^{1}(Q) \cong$ Z. The invariant $G(f)$ can now be defined by setting $G(f)=\bar{\varphi}^{*}(\eta) \in$ $H^{1}\left(S^{1}\right) \cong \mathbf{Z}$ where $\bar{\varphi}^{*}: H^{1}(Q) \rightarrow H^{1}\left(S^{1}\right)$ is the induced cohomology homomorphism as described in $\S 3$ and $\eta$ is a generator of $H^{1}(Q) \cong \mathbf{Z}$. The details of this definition are given in $\$ 7$.

3. Cohomology of set-valued maps. In this section we collect the facts concerning the cohomology of set-valued maps which we will need for the definition and invariance of $G(f)$. A set-valued map from a set $X$ into a set $Y$ is a function $F: X \rightarrow 2^{Y}$ which assigns to each point $x \in X$ a subset $F(x)$ of $Y, F(x) \subset Y$. The image of a set-valued map $F: X \rightarrow 2^{Y}$ is given by

$$
\operatorname{Im} F=\bigcup_{x \in X} F(x) \subset Y .
$$

We now describe a class of set-valued maps for which we can define an induced cohomology homomorphism. We say that a set-valued map $F$ : $X \rightarrow 2^{Y}$ is allowable if

1. $X$ and $Y$ are Hausdorff topological spaces,

2. $F(x)$ is nonempty and contractible for all $x \in X$, and

3. gr $F=\{(x, y) \in X \times Y \mid y \in F(x)\}$ is compact.

Note that conditions 2 and 3 imply that $X$ is compact. The definition of the induced cohomology homomorphism for an allowable set-valued map is based on the Vietoris-Begle mapping theorem. The following version is a special case of Theorem 15, p. 344 of Spanier [8].

TheOREM (VIETORIS-Begle). Let $\psi: X \rightarrow Y$ be a surjective continuous map between compact Hausdorff spaces. Let $\bar{H}^{*}$ denote Alexander cohomology theory with integer coefficients and let $\bar{H}^{*}$ denote the corresponding reduced theory. If $\bar{H}^{q}\left(\psi^{-1}(y)\right)=0$ for all $y \in Y$ and all $q$, then $\psi^{*}: \bar{H}^{q}(Y) \rightarrow \bar{H}^{q}(X)$ is an isomorphism for all $q$.

Let $F: X \rightarrow 2^{Y}$ be allowable and consider the following diagram where $i$ is the inclusion map, $P_{X}$ and $P_{Y}$ are the natural projection maps, and $P_{X}^{\prime}=$ $P_{X} \circ i$ and $P_{Y}^{\prime}=P_{Y} \circ i$ are the restrictions of $P_{X}$ and $P_{Y}$ to gr $F$. 


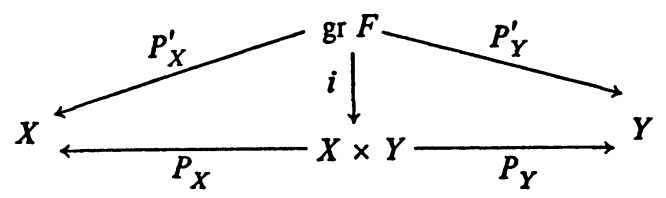

Since $P_{X}^{\prime-1}(x)=x \times F(x), P_{X}^{\prime}$ satisfies the hypothesis of the Vietoris-Begle theorem. Hence, it makes sense to define $F^{*}=P_{X}^{\prime *-1} \circ P_{Y}^{\prime *}: \bar{H}^{q}(Y) \rightarrow$ $\bar{H}^{q}(X)$ for all $q[3]$.

The following facts follow rather easily from this definition.

3.1. For single-valued maps. Suppose $F$ is single-valued, i.e., $F(x)=\{f(x)\}$ for some single-valued function $f: X \rightarrow Y$. Then $f$ is continuous and $F^{*}=f^{*}$ : $\bar{H}^{q}(Y) \rightarrow \bar{H}^{q}(X)$ for all $q$.

3.2. Composition with single-valued maps. Suppose $Z$ is a compact Hausdorff space, $g$ is a single-valued continuous function, $g: Z \rightarrow X$, and $F: X \rightarrow Y$ is an allowable set-valued map. Then $F \circ g: Z \rightarrow 2^{Y}, F \circ g(z)=F(g(z)) \subset Y$, is an allowable set-valued map and $(F \circ g)^{*}=g^{*} \circ F^{*}$. This follows from the diagrams below where the unmarked arrows are restrictions of the appropriate projections.
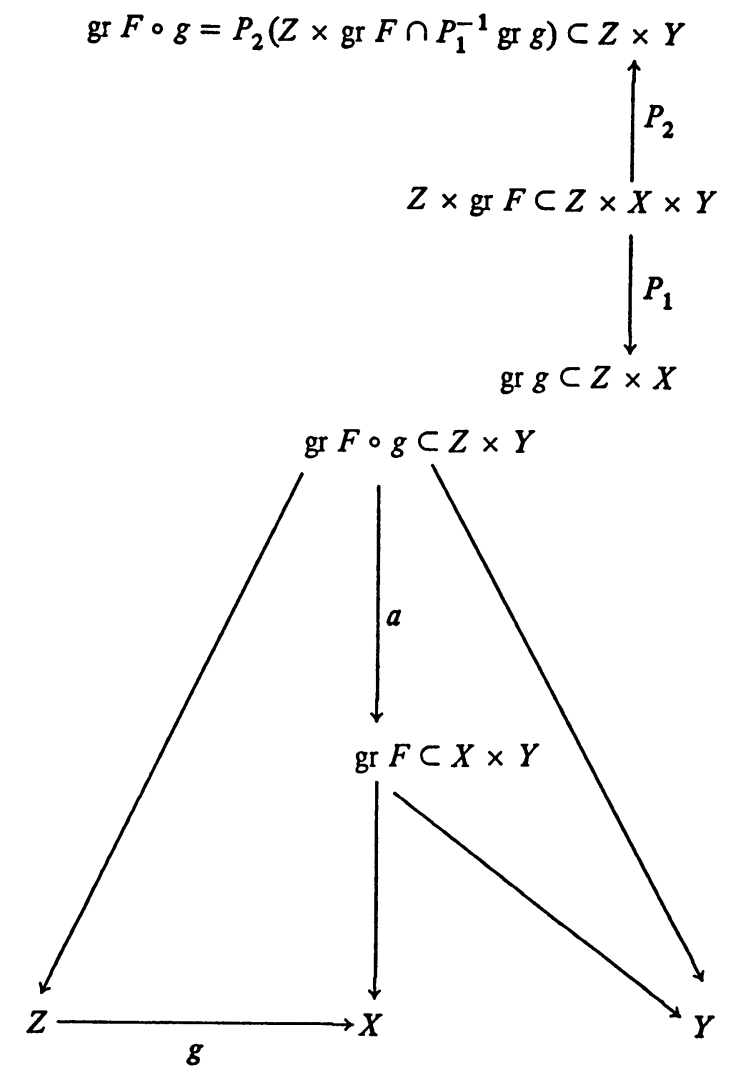


$$
a(z, y)=(g(z), y) \text { for all }(z, y) \in \operatorname{gr} F \circ g .
$$

3.3. Homotopy. By a homotopy between allowable set-valued maps $F, G$ : $X \rightarrow 2^{Y}$ we mean an allowable set-valued map $H: X \times I \rightarrow 2^{Y}$ such that $H \circ i_{0}=F$ and $H \circ i_{1}=G$, where $i_{0}, i_{1}: X \rightarrow I, i_{0}(x)=(x, 0)$ and $i_{1}(x)=$ $(x, 1)$ for all $x \in X$. Since $i_{0}$ is homotopic to $i_{1}, i_{0}^{*}=i_{1}^{*}$. It follows from property 2 above that $F^{*}=\left(H \circ i_{0}\right)^{*}=i_{0}^{*} \circ H^{*}=i_{1}^{*} \circ H^{*}=\left(H \circ i_{1}\right)^{*}=$ $G^{*}$. That is, homotopic allowable set-valued maps induce identical cohomology homomorphisms.

There is a special circumstance in which allowable set-valued maps are homotopic. If $F, G: X \rightarrow 2^{Y}$ are allowable set-valued maps and for each $x \in X, F(x) \subset G(x)$, then $F$ is homotopic to $G$. Indeed, a homotopy $H$ : $X \times I \rightarrow 2^{Y}$ from $F$ to $G$ may be defined by

$$
H(x, t)= \begin{cases}F(x) & \text { for } 0 \leqslant t<1, \\ G(x) & \text { for } t=1\end{cases}
$$

Note that gr $H=(\operatorname{gr} F \times I) \cup(\operatorname{gr} G \times\{1\}) \subset X \times Y \times I$ where we identify $X \times Y \times I$ with $X \times I \times Y$ in the obvious manner.

We will also have use for the following continuity property of allowable set-valued maps. The proof is easy and left to the reader.

3.4. LEMMA If $F: X \rightarrow 2^{Y}$ is an allowable set-valued map, then for each $x \in X$ and open set $V$ containing $F(x)$, there exists a neighborhood $U$ of $x$ such that $x^{\prime} \in U$ implies $F\left(x^{\prime}\right) \subset U$.

4. Geometry of flat immersions. In the following proposition we establish some properties of $V(p)$ which we will need for the definition and proof of invariance of $G(f)$ for $h \geqslant 1$.

4.1. Proposition. Given an isometric immersion $f: C_{h} \rightarrow \mathbf{R}^{3}$, let $V(p)$ be defined as in $\$ 2$.

(a) For each $p \in S^{1}, V(p)$ is a nonempty closed line segment.

(b) The graph of $V$ and the image of $V$ are compact.

(c) There exist monotone nondecreasing functions $T, B: \mathbf{R} \rightarrow \mathbf{R}$ such that $T(s+2 \pi)=T(s), B(s+2 \pi)=B(s), \frac{1}{2}(T(s)+B(s))=s$, and $(T(s)-s)$ $\in \tilde{V}(s)$ for all $s \in \mathbf{R}$.

(c') If we are given $\alpha, \beta \in \mathbf{R}$ and $t \in \tilde{V}(\alpha)$ and $t^{\prime} \in \tilde{V}(\beta)$ such that the segment from $(\alpha-t,-h)$ to $(\alpha+t, h)$ does not cross the segment from $\left(\beta-t^{\prime},-h\right)$ to $\left(\beta+t^{\prime}, h\right)$, then we may require $T(\alpha)-\alpha=t$ and $T(\beta)-$ $\beta=t^{\prime}$.

Proof. Let $A=\{s \in \mathbf{R} \mid$ mean curvature of $g$ at $(s, 0) \neq 0\}$. The following facts about isometric immersions of flat surfaces are well known [2], [5], [9]. Through each interior point of nonzero mean curvature there exists a unique 
straight line segment which lies in the surface. This line segment, when extended in either direction, continues to lie in the surface indefinitely or until it hits the boundary. Furthermore the mean curvature is nonzero at all points on such lines.

We apply these facts to our isometric immersion $g: \tilde{C}_{h} \rightarrow \mathbf{R}^{3}$. For $s \in A$ we get such a straight line segment through $(s, 0)$. This line segment cannot be horizontal for then it would extend to be the whole $s$-axis $=\{(s, 0) \mid s \in \mathbf{R}\}$. But $g(2 \pi)=g(0)$, a contradiction. Hence this segment extends to form a line segment going from a point $(B(s),-h)$ on the bottom boundary to a point $(T(s), h)$ on the top boundary. Note that if $t \in \tilde{V}(s)$ then the segment from $(s-t,-h)$ to $(s+t, h)$ in $\tilde{C}_{h}$ is mapped rigidly by $g$. From the uniqueness mentioned in the above paragraph it follows that, for $s \in A, \tilde{V}(s)$ consists of a single point, $\tilde{V}(s)=\{T(s)-s\}$.

Now consider an $s \in$ bdry $A$. Then there exist points $s_{n} \in A, s_{n} \rightarrow s$. We may arrange things so that the sequence $s_{n}$ approaches $s$ from one side and monotonically. We may assume without loss of generality that $s_{n} \uparrow s$. We must have $T\left(s_{n}\right)$ and $B\left(s_{n}\right)$ both monotonically increasing to avoid having the straight line segments associated with $\left(s_{n}, 0\right)$ and $\left(s_{n+1}, 0\right)$ cross each other. (Such crossings are ruled out by the properties of flat surfaces mentioned above.) For the same reason there are upper bounds on $T\left(s_{n}\right)$ and $B\left(s_{n}\right)$. Hence $T_{0}=\lim _{n \rightarrow \infty} T\left(s_{n}\right)$ and $B_{0}=\lim _{n \rightarrow \infty} B\left(s_{n}\right)$ exist. Since $\| g\left(T\left(s_{n}\right), h\right)-$ $g\left(B\left(s_{n}\right),-h\right)\|=\|\left(T\left(s_{n}\right), h\right)-\left(B\left(s_{n}\right),-h\right) \|$ and $\frac{1}{2}\left(T\left(s_{n}\right)+B\left(s_{n}\right)\right)=s_{n}$ for all $n$, it follows that

$$
\left\|g\left(T_{0}, h\right)-g\left(B_{0},-h\right)\right\|=\left\|\left(T_{0}, h\right)-\left(B_{0},-h\right)\right\|
$$

and

$$
\frac{1}{2}\left(T_{0}+B_{0}\right)=s .
$$

Hence the whole segment from $\left(B_{0},-h\right)$ to $\left(T_{0}, h\right)$ in $\tilde{C}_{h}$ is mapped rigidly by g.

It follows that $T_{0}-s \in \tilde{V}(s)$. Also, to avoid crossing straight line segments we must have $\tilde{V}(s)=\left\{T_{0}-s\right\}=\left\{s-B_{0}\right\}$. Set $T(s)=T_{0}$ and $B(s)$ $=B_{0}$.

Set $B=\mathbf{R}-\operatorname{cl} A$, where $\mathrm{cl} A$ denotes the closure of $A$. Since $B$ is open it is the union of disjoint open intervals. Let $(a, b)$ be one of those intervals. Since the mean curvature of $g$ at each point $(s, 0)$ is zero for $s \in(a, b)$, the curvature of the curve $g \mid \mathbf{R} \times 0$ is zero for all $s \in(a, b)$. Hence $g$ maps $[a$, $b] \times 0 \subset \tilde{C}_{h}$ rigidly. Since $g$ is periodic, it follows that the segment $(a, b)$ is not infinite. Since $a, b \in \mathrm{cl} A, T(a), T(b), B(a)$ and $B(b)$ are defined and $g$ maps rigidly the segments from $(B(a),-h)$ to $(T(a), h)$ and from $(B(b),-h)$ to $(T(b), h)$. It is now an exercise in elementary geometry to show that $g$ must map rigidly the whole quadrilateral whose vertices are $(B(a),-h),(T(a), h)$, 
$(T(b), h)$ and $(B(b),-h)$. Now it is clear that $\tilde{V}(s)$ is a closed line segment for each $s \in(a, b)$. For $\tau \in(0,1)$ define

$$
T(\tau b+(1-\tau) a)=\tau T(b)+(1-\tau) T(a)
$$

and

$$
B(\tau b+(1-\tau) a)=\tau B(b)+(1-\tau) B(a) .
$$

Conclusions (a) and (c) are now easily verified. Conclusion $\left(c^{\prime}\right)$ takes a slight modification in the definitions of $T$ and $B$ which we leave to the reader.

Now we establish conclusion (b). Recall that the graph of $V$ is given by gr $V=\left\{(p, t) \in S^{1} \times \mathbf{R} \mid t \in V(p)\right\}$. We will show that gr $V \subset S^{1} \times[-r, r]$ for some $r$ and that gr $V$ is closed in $S^{1} \times \mathbf{R}$. Clearly the compactness of gr $V$ follows from these two conditions. Suppose there were no $r$ such that

$$
\text { gr } V \subset S^{1} \times[-r, r] \text {. }
$$

Then there exist $p \in S^{1}$ and $t \in V(p)$ with $|t|$ arbitrarily large. But $C_{h}$ is compact and hence $g\left(C_{h}\right)$ is bounded and yet

$$
\|g(s+t, h)-g(s-t,-h)\|=\|(s+t, h)-(s-t,-h)\| \geqslant|2 t|,
$$

where $\pi(s, 0)=(p, 0)$, a contradiction. The fact that gr $V$ is closed follows in a straightforward manner from the definitions.

The image of $V=\left\{t \in \mathbf{R} \mid t \in V(p)\right.$ for some $\left.p \in S^{1}\right\}$ is simply the image of gr $V$ under the projection $S^{1} \times \mathbf{R} \rightarrow \mathbf{R},(s, t) \rightarrow t$, and is consequently compact. Q.E.D.

From the proof we have the following corollary.

4.2. Corollary. Suppose $H: C_{h} \times I \rightarrow \mathbf{R}^{3}$ is a homotopy through isometric immersions and $V_{H}: C_{h} \times I \rightarrow 2^{\mathbf{R}}$ is defined by $V_{H}(p, \tau)=V_{H_{\tau}}(p)$ where $H_{\tau}$ : $C_{h} \rightarrow \mathbf{R}^{3}$ is the isometric immersion given by $H_{\tau}(p)=H(p, \tau)$ for $p \in C_{h}$ and $\tau \in I$. Then the graph of $V_{H}$ and the image of $V_{H}$ are compact.

5. A crucial calculation. With the aid of Proposition 4.1(c) and ( $\left.c^{\prime}\right)$ we will prove the following key proposition.

5.1. Proposition. Given $\alpha, \beta \in \mathbf{R}, \alpha<\beta, t \in \tilde{V}(\alpha)$, and $t^{\prime} \in \tilde{V}(\beta)$ such that $g(\alpha+t, h)-g(\alpha-t,-h)=\lambda\left(g\left(\beta+t^{\prime}, h\right)-g\left(\beta-t^{\prime}, h\right)\right)$ for some $\lambda<0$; then $\beta-\alpha>\pi h$.

Proof. Let $T$ and $B$ be given by Proposition 4.1(c) and ( $\left.c^{\prime}\right)$. The hypothesis for $\left(c^{\prime}\right)$ holds because the crossing of the line segments is incompatible with the antiparallel hypothesis in the present proposition and the fact that $g$ is an immersion.

We first prove the proposition under the additional hypothesis that $T$ and $B$ are continuously differentiable.

Define $\tilde{T}, \tilde{B}: \mathbf{R} \rightarrow \mathbf{R}^{3}$ by 


$$
\tilde{T}(s)=g(T(s), h) \text { and } \tilde{B}(s)=g(B(s),-h) .
$$

Set $\varphi(s)=\tilde{T}(s)-\tilde{B}(s)$. Then

$$
\begin{aligned}
\|\varphi(s)\| & =\|(T(s), h)-(B(s),-h)\| \\
& =\left((2 h)^{2}+(T(s)-B(s))^{2}\right)^{1 / 2} \geqslant 2 h
\end{aligned}
$$

for all $s \in \mathbf{R}$. Also $\varphi(\beta)=\lambda \varphi(\alpha)$ with $\lambda<0$. Thus $\varphi(s)$ is a curve lying outside an open ball of radius $2 h$ which starts at a point on one central ray and ends at a point on the opposite central ray. Hence, the length of $\varphi:[\alpha$, $\beta] \rightarrow \mathbf{R}^{3}$ is at least $2 \pi h$. We now calculate

$$
\begin{aligned}
2 \pi h & <\int_{\alpha}^{\beta}\left\|\varphi^{\prime}(s)\right\| d s=\int_{\alpha}^{\beta}\left\|\tilde{T}^{\prime}(s)-\tilde{B}^{\prime}(s)\right\| d s \\
& <\int_{\alpha}^{\beta}\left\|\tilde{T}^{\prime}(s)\right\| d s+\int_{\alpha}^{\beta}\left\|\tilde{B}^{\prime}(s)\right\| d s \\
& =\left[\left(\beta+t^{\prime}\right)-(\alpha+t)\right]+\left[\left(\beta-t^{\prime}\right)-(\alpha-t)\right]
\end{aligned}
$$

because $g$ is an isometric immersion and the curves $s \rightarrow(T(s), h) \in \tilde{C}_{h}$ and $s \rightarrow(B(s),-h) \in \tilde{C}_{h}$ clearly have lengths $\left(\beta+t^{\prime}\right)-(\alpha+t)$ and $\left(\beta-t^{\prime}\right)-$ $(\alpha-t)$. Hence $\beta-\alpha>\pi h$.

The inequality $\beta-\alpha>\pi h$ is established without the additional differentiability hypothesis by mimicking the above argument and replacing integrals of norms of derivatives by limits of finite sums of norms of differences.

Consider the possibility $\beta-\alpha=\pi h$. Then in the above calculation we must have $\|\varphi(s)\|=2 h$ for all $s$. Consequently, $T(s)=s$ and $B(s)=s$ for all $s$. Thus $T$ and $B$ are continuously differentiable. Where the triangular inequality was used above we must have equality and hence $\tilde{T}^{\prime}(s)=$ $k\left(-\tilde{B}^{\prime}(s)\right)$ for some $k \geqslant 0$. Since $\tilde{T}^{\prime}(s)=\partial g(s, h) / \partial s$ and $g$ is an isometric immersion we have $\left\|\tilde{T}^{\prime}(s)\right\|=1$ for all $s$. Similarly $\left\|\tilde{B}^{\prime}(s)\right\|=1$, and so $k=1$ and $\dot{T}^{\prime}(s)=-\tilde{B}^{\prime}(s)$. But now we have $g(s, 0)=\frac{1}{2}(\check{T}(s)+\tilde{B}(s))$ and $\partial g(s, 0) / \partial s=0$ contradicting the fact that $g$ is an isometric immersion. Therefore $\beta-\alpha>\pi h$ as we wished to show. Q.E.D.

The following corollary follows easily from the above proof. Alternately one can derive it from the statement of Proposition 5.1 by considering the double covering of $M_{l}$ by a cylinder.

5.2. Corollary. If $M_{l}$ isometrically immerses in $\mathbf{R}^{3}$, then $l>\pi / 2$.

6. The space $Q$. Consider $\mathbf{R}^{2}=\mathbf{R}^{2} \times 0 \subset \mathbf{R}^{2} \times \mathbf{R}^{1}=\mathbf{R}^{3}$ and so $S^{1} \subset \mathbf{R}^{2} \subset$ $\mathbf{R}^{3}$. Now embed $S^{1}$ in $Q$ by the map $j: S^{1} \rightarrow Q$ given by $j(p)=\left(e_{3}, p\right)$ for all $p \in S^{1}$.

6.1. LeMMA. $j\left(S^{1}\right)$ is a strong deformation retract of $A$. 
Proof. First apply Gram-Schmidt to get a homotopy which moves ( $u$, $v) \in Q$ over to $\left(u /\|u\|, v^{1}\right)$. Then rotate $\left(u /\|u\|, v^{1}\right)$ by the rotation which leaves the line perpendicular to $u$ and $e_{3}$ fixed and moves $u /\|u\|$ along the smaller geodesic arc on $S^{2}$ from $u /\|u\|$ to $e_{3}$. Q.E.D.

7. Definition of $G(f)$ for $h \geqslant 1$. Let $f: C_{h} \rightarrow \mathbf{R}^{3}$ be an isometric immersion with $h \geqslant 1$. Let $T, g, C_{h}, \pi, \tilde{V}, V, \tilde{W}, W, R$ and $Q$ be as defined in $\S 2$. We will define $\bar{\varphi}$ more carefully here. First set $\varphi(p)=(W(p), T(p))=\{(w, T(p)) \in$ $\left.\mathbf{R}^{3} \times \mathbf{R}^{3} \mid w \in W(p)\right\}$ for each $p \in S^{1}$. Next pick a $w \in \tilde{W}(0)$ and a rotation $U \in S O(3)$ such that $U(w)=\|w\| e_{3}$ where $e_{3}=(0,0,1)$ and $\|\cdot\|$ is the Euclidean norm. Now set $\bar{\varphi}(p)=U(\varphi(p))$ for each $p \in S^{1}$ where

$$
U(x, y)=(U(x), U(y)) \text { for all }(x, y) \in \mathbf{R}^{3} \times \mathbf{R}^{3} \text {. }
$$

In light of Proposition 5.1, this gives a set-valued map $\bar{\varphi}: S^{1} \rightarrow 2^{Q}$.

Next, we will use the results of $\S 4$ to show that $\bar{\varphi}$ is allowable. The space $S^{1}$ and $Q$ are clearly Hausdorff and so condition 1 of the definition of allowable is satisfied. By Proposition 4.1(a), for each $p \in S^{1}, V(p)$ is a nonempty closed line segment. Hence $W(p)=\tilde{W}(s)=\left.d g\right|_{(s, 0)} V(p) \times h$, where $\pi(s, 0)=(p, 0)$ is also a nonempty closed line segment. Therefore the same is true of $\bar{\varphi}(p)=U(\varphi(p))=U(W(p), T(p))$. This establishes condition 2 in the definition of allowable. It is a straightforward exercise in the definition of $\bar{\varphi}$ to express the graph of $\bar{\varphi}$ as a continuous image of the graph of $V$. Hence, by Proposition 4.1(b), gr $\bar{\varphi}$ is compact. Thus $\bar{\varphi}$ is allowable.

7.0. Remark. Note that $\varphi: S^{1} \rightarrow 2^{\mathbf{R}^{3} \times \mathbf{R}^{3}}$ is also allowable.

According to $\S 3$, there is a well-defined induced homomorphism $\bar{\varphi}^{*}$ : $\bar{H}^{1}(Q) \rightarrow \bar{H}^{1}\left(S^{1}\right)$. It follows from Proposition 6.1 that $j^{*}: \bar{H}^{1}(Q) \rightarrow \bar{H}^{1}\left(S^{1}\right)$ is an isomorphism. Let $\eta$ be a generator of $\bar{H}^{1}\left(S^{1}\right) \cong \mathbf{Z}$. Define $G(f) \in \mathbf{Z}$ by the requirement

$$
\bar{\varphi}^{*} \circ j^{*-1}(\eta)=G(f) \eta .
$$

It is clear from (*) that $G(f)$ does not depend on the choice of generator $\eta$. The next two lemmas complete the demonstration that $G(f)$ is well defined by showing that $G(f)$ does not depend on the choices of $U$ or $w$.

7.1. Lemma. $G(f)$ does not depend on the choice of $U \in S O(3)$.

Proof. Let $U^{\prime} \in S O(3)$ be another rotation such that $U^{\prime}(w)=\|w\| e_{3}$ and set $\bar{\varphi}^{\prime}(p)=U^{\prime}(\varphi(p))$ for $p \in S^{1}$. The subset $F$ of $S O(3)$ consisting of rotations $\bar{U}$ such that $\bar{U}(w)=\|w\| e_{3}$ is homeomorphic to $S O(2) \cong S^{1}$ and hence there is a path $\gamma:[0,1] \rightarrow F$ such that $\gamma(0)=U$ and $\gamma(1)=U^{\prime}$. Using Proposition 5.1 and Corollary 4.2 we see that this gives rise to a homotopy.

$$
\varphi_{t}(p)=\gamma(t)(\varphi(p)) \text { from } \bar{\varphi} \text { to } \bar{\varphi}^{\prime} .
$$

Hence, by $\S 3, \bar{\varphi}^{*}=\bar{\varphi}^{\prime *}$ and so $G(f)$ does not depend on the choice of $U$. Q.E.D. 
7.2. Lemma. $G(f)$ does not depend on the choice of $w \in \tilde{W}(0)$.

Proof. Let $w^{\prime} \in \tilde{W}(0)$ be another choice. We know by Proposition 4.1(a) that $\tilde{W}(0)$ is a closed line segment. Since $\left.d g\right|_{(0,0)}$ is isometric and hence 1-1 we conclude that $0 \notin \tilde{W}(0)$. Hence it is possible to find a curve $\gamma(t) \in S O(3)$ such that $\gamma(0)=U$ and $\gamma(t)\left(w_{t}\right)=\left\|w_{t}\right\| e_{3}$ where $w_{t}=t w^{\prime}+(1-t) w$. It follows from Proposition 5.1 that $\varphi_{t}(p)=\gamma(t)(\varphi(p)) \subset Q$ for all $(p, t) \in S^{1}$ $\times I$. It now follows from $\S 3$ that $(G(f)$ calculated with $w$ and $U)=(G(f)$ calculated with $w^{\prime}$ and $\left.\varphi(1)\right)$. Q.E.D.

The following theorem follows immediately from the definition of $G(f)$.

7.3. THEOREM. If $f: C_{h} \rightarrow \mathbf{R}^{3}$ is an isometric immersion, $h \geqslant 1$, and $f$ is of the form $f=\gamma \times j: S^{1} \times I_{h} \rightarrow \mathbf{R}^{2} \times \mathbf{R}^{1}=\mathbf{R}^{3}$, where $\gamma: S^{2} \rightarrow \mathbf{R}^{2}$ is a smooth closed curve and $j: I_{h} \rightarrow \mathbf{R}^{1}$ is the inclusion map, then $G(f)=$ the tangent winding number of $\gamma$.

8. Invariance of $G(f)$. We continue in the context of $\S 7$. The next theorem states that for $f^{\prime}$ "close" to $f, G\left(f^{\prime}\right)=G(f)$. The nature in which $f^{\prime}$ is required to be close to $f$ is " $C$ " closeness" for $x \in C_{h}$ plus " $C$ l closeness" for $x \in S^{1} \times 0 \subset S^{1} \times I_{n}=C_{h}$. The invariance of $G(f)$ under isometric homotopy is an immediate corollary of this result.

The straightforward approach to showing that $G(f)$ is invariant under isometric homotopy is to try to obtain a homotopy of $\bar{\varphi}$ from an isometric homotopy of $f$. There is a hitch to this approach. $\bar{\varphi}$ depends on a choice of $U \in S O$ (3) and it may not be possible to choose $U$ as a continuous function of the homotopy parameter. The following proof is one of at least two ways to get around this problem.

8.1. THEOREM. Given an isometric immersion $f: C_{h} \rightarrow \mathbf{R}^{3}$ with $h \geqslant 1$, there exists a $\delta>0$ such that $G\left(f^{\prime}\right)=G(f)$ for all isometric immersions $f^{\prime}: C_{h} \rightarrow \mathbf{R}^{3}$ with $\rho\left(f, f^{\prime}\right)<\delta$, where

$$
\rho\left(f, f^{\prime}\right)=\sup _{x \in C_{h}}\left\|f(x)-f^{\prime}(x)\right\|+\sup _{s \in \mathbf{R}}\left\|\frac{d}{d s}\left(g(s, 0)-g^{\prime}(s, 0)\right)\right\| .
$$

We will need several lemmas for the proof. We make use of the following notation. If $(X, d)$ is a metric space, $A \subset X$, and $\varepsilon>0$, then $B_{\varepsilon}(A)$ denotes $\{x \in X \mid d(x, a)<\varepsilon$ for some $a \in A\}$. Also, let $\mathbf{R}^{+}$denote $\{t \in \mathbf{R} \mid t \geqslant 0\}$.

8.2. LemMA. Given a compact space $A$, a metric space $(B, d)$, an open subset $U \subset B$, and continuous maps $L: A \rightarrow B$ and $T: A \rightarrow \mathbf{R}^{+}$such that $L\left(T^{-1}(0)\right)$ $\subset U$, then there exists an $\varepsilon>0$ such that if $\bar{L}: A \rightarrow B$ and $\bar{T}: A \rightarrow \mathbf{R}^{+}$satisfy $d(\bar{L}(a), L(a))<\varepsilon$ and $|\bar{T}(a)-T(a)|<\varepsilon$ for all $a \in A$, then $\bar{L}\left(\bar{T}^{-1}(0)\right) \subset$ $U$. 
Proof. By compactness we can find an $\epsilon_{1}>0$ such that $B_{2 \epsilon_{1}} L\left(T^{-1}(0)\right)$ $\subset U$. Since $T$ is strictly positive on the compact set $V=A-L^{-1}\left(B_{\epsilon_{1}}\left(L\left(T^{-1}(0)\right)\right)\right)$, there is an $\epsilon_{2}>0$ such that $T(v) \geqslant \epsilon_{2}$ for all $v \in V$. Then $\epsilon=\min \left(\epsilon_{1}, \epsilon_{2}\right)$ satisfies the conclusion of the lemma. Q.E.D.

8.3. LEMMA. If $f: C_{h} \rightarrow \mathbf{R}^{3}$ is an isometric immersion such that gr $\varphi_{f} \subset M$ with $M$ open, then there exists a $\delta>0$ such that gr $\varphi_{f^{\prime}} \subset M$ for all isometric immersions $f^{\prime}: C_{h} \rightarrow \mathbf{R}^{3}$ with $\rho\left(f, f^{\prime}\right)<\delta$.

Proof. In order to make use of compactness we first show that $V_{f}(p) \subset$ $[-\pi, \pi]$ for all isometric immersions and all $p \in S^{1}$. Let $t \in V_{f}(p)$. Then, with $\pi(s, 0)=(p, 0)$,

$$
\begin{aligned}
t^{2}+h^{2} & =\|(t, h)\|^{2}=\|g(s+t, h)-g(s, 0)\|^{2} \\
& =\|f(\pi(s+t, h))-f(\pi(s, 0))\|^{2} \leqslant\|(\pi, h)\|^{2} \leqslant \pi^{2}+h^{2}
\end{aligned}
$$

since $f$ is an isometric immersion and the distance between any point $x \in C_{h}=S^{1} \times I_{h}$ and $y \in S^{1} \times 0 \subset C_{h}$ is at most $\|(\pi, h)\|$. Hence $|t| \leqslant \pi$, and so $V_{f}(p) \subset[-\pi, \pi]$ as we wished to show.

Now apply Lemma 8.2 with $A=S^{1} \times[-\pi, \pi], B=S^{1} \times \mathbf{R}^{3} \times \mathbf{R}^{3}, U=$ $M, T=k_{f}, \bar{T}=k_{f}, L=\theta_{f}$, and $\bar{L}=\theta_{f}$, where

$$
k_{f}(p, t)=|\|g(s+t, h)-g(s-t,-h)\|-\|(s+t, h)-(s-t,-h)\||
$$

and

$$
\theta_{f}(p, t)=(p, g(s+t, h)-g(s, 0), T(p))
$$

for $(p, t) \in S^{1} \times[-\pi, \pi]$, with $\pi(s, 0)=(p, 0)$. Q.E.D.

An analogous argument proves

8.4. LEMMA. Given an isometric immersion $f: C_{h} \rightarrow \mathbf{R}^{3}$, for every $\varepsilon>0$ there exists a $\delta>0$ such that $\rho\left(f, f^{\prime}\right)<\delta$ implies $\tilde{W}_{f^{\prime}}(0) \subset B_{\varepsilon} \tilde{W}_{f}(0)$, for all isometric immersions $f^{\prime}$.

8.5. LEMMA. Given an isometric immersion $f: C_{h} \rightarrow \mathbf{R}^{\mathbf{3}}$, there exists an $\varepsilon>0$ such that $U\left(\operatorname{cl~} B_{\varepsilon} \operatorname{Im} \varphi_{f}\right) \subset Q$ for all $U \in S O(3)$ such that there exists a $w \in \tilde{W}_{f}(0)$ with $U(w /\|w\|)=e_{3}$ (i.e., for all $U \in S O(3)$ that can be used to define $\bar{\varphi})$.

Proof. Set $Z=\left\{U \in S O(3) \mid U(w /\|w\|)=e_{3}\right.$ for some $\left.w \in \tilde{W}_{f}(0)\right\}$. Using the compactness of $\tilde{W}_{f}(0)$ it is easy to show that $Z$ must be closed in $S O$ (3) and hence compact. Consider the map $L: S O(3) \times \mathbf{R}^{3} \times \mathbf{R}^{\mathbf{3}} \rightarrow \mathbf{R}^{\mathbf{3}} \times$ $\mathbf{R}^{3}$ given by $L(U, x, y)=(U x, U y)$. By the definition of $Z$ and Proposition 5.1 we have $L\left(Z \times \operatorname{Im} \varphi_{f}\right) \subset Q$. Since $Q$ is open in $\mathbf{R}^{3} \times \mathbf{R}^{3}$ and both $Z$ and $\operatorname{Im} \varphi_{f}$ are compact, there exists an $\varepsilon>0$ such that $L\left(Z \times \operatorname{cl} B_{\varepsilon} \operatorname{Im} \varphi_{f}\right) \subset Q$. The lemma now follows from the definition of $L$. Q.E.D. 
Proof of TheOREM 8.1. Given an isometric immersion $f: C_{h} \rightarrow \mathbf{R}^{3}$, we will construct an allowable, piecewise constant, set-valued map $\bar{\psi}: S^{1} \rightarrow 2^{Q}$ such that $\bar{\varphi}_{f}(x) \subset \bar{\psi}(x)$ and $\bar{\varphi}_{f^{\prime}}(x) \subset \bar{\psi}(x)$ for all $x \in S^{1}$ and $f^{\prime}$ sufficiently close to $f$. To do this, we first construct an allowable piecewise constant set-valued map $\psi: S^{1} \rightarrow 2^{\mathbf{R}^{3} \times \mathbf{R}^{3}}$ such that $\operatorname{gr} \varphi_{f} \subset$ int $\operatorname{gr} \psi$.

Let $\varepsilon$ satisfy Lemma 8.5 .

Let $\bar{\pi}: \mathbf{R} \rightarrow S^{1}$ be the covering map given by $\bar{\pi}(t)=(\cos t, \sin t)$. Let $d$ be the arc length metric on $S^{1}$, i.e., $d(p, q)=\min \{|s-t| \mid \bar{\pi}(s)=p$ and $\bar{\pi}(t)=$ q). Applying Lemma 3.4 and Remark 7.0 to $\varphi_{f}: S^{1} \rightarrow 2^{\mathbf{R}^{3} \times \mathbf{R}^{3}}$ we obtain for each $p \in S^{1}$ a $\delta(\mathrm{p})>0$ such that $d(p, q)<\delta(p)$ implies $\varphi_{f}(q) \subset B_{\varepsilon} \varphi_{f}(p)$. Let $\alpha$ be a Lebesgue number for the open cover $\left\{B_{\delta(p)}(p) \subset S^{1} \mid p \in S^{1}\right\}$ of $S^{1}$. Choose a sequence $0=s_{0}<s_{1}<\cdots<s_{n}=2 \pi$ such that $s_{i+1}-s_{i}<$ $\alpha / 2$. Set $s_{n+1}=s_{1}+2 \pi$.

For each $i=1, \ldots, n$ there exists a $p_{i}$ such that $\bar{\pi}\left[s_{i-1}, s_{i+1}\right] \subset B_{\delta\left(p_{i}\right)}\left(p_{i}\right) \subset$ $S^{1}$. Set $\varphi_{i}=\varphi_{f}\left(p_{i}\right)$ for $1 \leqslant i \leqslant n$ and set $\varphi_{0}=\varphi_{n}$. We now define $\psi$ : $S^{1} \rightarrow 2^{\mathrm{R}^{3} \times \mathrm{R}^{3}}$ by setting

$$
\psi(\bar{\pi}(s))= \begin{cases}\operatorname{cl} B_{\varepsilon} \varphi_{i} \cap \operatorname{cl} B_{\varepsilon} \varphi_{i+1} & \text { if } s \in\left(s_{i}, s_{i+1}\right), 0 \leqslant i<n, \\ \operatorname{cl} B_{\varepsilon} \varphi_{i} & \text { if } s=s_{i}, 0 \leqslant i \leqslant n,\end{cases}
$$

for $s \in[0,2 \pi]$.

It is easy to see that $\mathrm{gr} \psi$ is compact because

$$
\begin{aligned}
\operatorname{gr} \psi= & \bigcup_{i=0}^{n-1} \pi\left[s_{i}, s_{i+1}\right] \times \operatorname{cl} B_{\varepsilon} \varphi_{i} \cap \operatorname{cl} B_{\varepsilon} \varphi_{i+1} \\
& \cup \bigcup_{i=0}^{n}\left\{\bar{\pi}\left(s_{i}\right)\right\} \times \operatorname{cl} B_{\varepsilon} \varphi_{i} .
\end{aligned}
$$

Since $\varphi_{f}(p)$ is convex, $\dot{\psi}(p)$ is also convex for each $p \in S^{1}$. Hence $\psi$ is allowable.

It is easy to see that gr $\varphi_{f} \subset$ int gr $\psi$ because

$$
\begin{aligned}
\operatorname{gr} \varphi_{f} & \subset \bigcup_{i=0}^{n-1} \bar{\pi}\left(s_{i}, s_{i+1}\right) \times B_{\varepsilon} \varphi_{i} \cap B_{\varepsilon} \varphi_{i+1} \\
& \cup \bigcup_{i=1}^{n} \bar{\pi}\left(s_{i-1}, s_{i+1}\right) \times B_{\varepsilon} \varphi_{i-1} \cap B_{\varepsilon} \varphi_{i} \cap B_{\varepsilon} \varphi_{i+1} \\
& \subset \operatorname{gr} \psi .
\end{aligned}
$$

Since gr $\varphi_{f}$ is compact and gr $\varphi_{f} \subset$ int gr $\psi$, there exists an $\varepsilon^{\prime}>0$ such that $B_{\lambda} \operatorname{gr} \varphi_{f} \subset$ int $g r \psi$ where $\lambda=\varepsilon^{\prime}\left(\varepsilon^{\prime}+\operatorname{diam}\left(\operatorname{Im} \varphi_{f} \cup\{(0,0)\}\right)\right)+\varepsilon^{\prime}$, and where we are using the maximum metric on the product space $S^{1} \times\left(\mathbf{R}^{3} \times \mathbf{R}^{3}\right)$. From Lemma 8.3 we obtain a $\delta_{1}>0$ such that if $f^{\prime}: C_{h} \rightarrow \mathbf{R}^{3}$ is an isometric 
immersion and $\rho\left(f, f^{\prime}\right)<\delta_{1}$, then gr $\varphi_{f^{\prime}} \subset B_{\varepsilon^{\prime}}$ gr $\varphi_{f}$. In particular, for such $f^{\prime \prime}$ s we have $\varphi_{f^{\prime}}(p) \subset B_{\varepsilon^{\prime}} \varphi_{f}(p)$ for all $p \in S^{1}$.

Next, by Lemma 8.4 there exists a $\delta_{2}>0$ such that if $f^{\prime}$ is an isometric immersion and $\rho\left(f, f^{\prime}\right)<\delta_{2}$, then $\tilde{W}_{f^{\prime}}(0) \subset B_{\varepsilon^{\prime}} \tilde{W}_{f}(0)$. Set $\delta=\min \left(\delta_{1}, \delta_{2}\right)$ and suppose $f^{\prime}$ is an isometric immersion such that $\rho\left(f, f^{\prime}\right)<\delta$. Pick a $w^{\prime} \in$ $\tilde{W}_{f^{\prime}}(0)$. Then there exists a $w \in \tilde{W}_{f}(0)$ such that $\left\|w-w^{\prime}\right\|<\varepsilon^{\prime}$. From $\|w\| \geqslant$ $h \geqslant 1$ and $\left\|w^{\prime}\right\| \geqslant h \geqslant 1$ it is not hard to show that $\|w /\| w\|-w /\| w^{\prime}\|\| \leqslant$ $\left\|w-w^{\prime}\right\|<\varepsilon^{\prime}$. Thus, we can find a $U^{\prime \prime} \in S O(3)$ such that $U^{\prime \prime}\left(w^{\prime} /\left\|w^{\prime}\right\|\right)=$ $w /\|w\|$ and $\sup _{\|v\|=1}\left\|U^{\prime \prime} v-v\right\|=\|w /\| w\left\|-w^{\prime} /\right\| w\|\|<\varepsilon^{\prime}$. Pick a $U \in$ $S O(3)$ such that $U(w /\|w\|)=e_{3}$. Set $U^{\prime}=U \circ U^{\prime \prime}$. We may then calculate $G(f)$ and $G\left(f^{\prime}\right)$ from $\bar{\varphi}_{f}=U \circ \varphi_{f}$ and $\bar{\varphi}_{f^{\prime}}=U^{\prime} \circ \varphi_{f^{\prime}}$ respectively.

Because $\varepsilon$ was chosen to satisfy Lemma 8.5 we have $U\left(\operatorname{cl} B_{\varepsilon} \operatorname{Im} \varphi_{f}\right) \subset Q$. But, from the definition of $\psi$ it follows that $\operatorname{Im} \psi \subset \operatorname{cl} B_{\varepsilon} \operatorname{Im} \varphi_{f}$. Set $\bar{\psi}=U \circ \psi$. Them $\operatorname{Im} \bar{\psi} \subset Q$. Since $\psi$ is allowable and $(x, y) \rightarrow(U x, U y)$ is a homeomorphism of $\mathbf{R}^{3} \times \mathbf{R}^{3}$ with itself, it follows that $\bar{\psi}=U \circ \psi$ is allowable. Hence $\bar{\psi}: S^{1} \rightarrow 2^{Q}$ is a well-defined allowable set-valued map.

Finally we observe that

$$
\begin{aligned}
\bar{\varphi}_{f^{\prime}}(p) & =U^{\prime} \circ \varphi_{f^{\prime}}(p)=U \circ U^{\prime \prime} \circ \varphi_{f^{\prime}}(p) \subset U \circ U^{\prime \prime} B_{\varepsilon^{\prime}} \varphi_{f}(p) \\
& \subset U\left(B_{\lambda} \varphi_{f}(p)\right) \subset U(\psi(p))=\bar{\psi}(p)
\end{aligned}
$$

for all $p \in S^{1}$. Since $f^{\prime}$ may be particularized to $f$ we also have $\bar{\varphi}_{f}(p) \subset \bar{\psi}(p)$ for all $p \in S^{1}$. Now by observation 3.3 we have $\bar{\varphi}_{f}$ is homotopic to $\bar{\psi}$ which is homotopic to $\bar{\varphi}_{f}$ and so $\bar{\varphi}_{f^{\prime}}^{*}=\bar{\varphi}_{f}^{*}$. Thus $G\left(f^{\prime}\right)=G(f)$ and the proof is complete. Q.E.D.

Combining Theorems 2.1, 2.2, 7.3 and 8.1 we have

8.6. Proposition. Given two isometric immersions of the form $\gamma \times j, \gamma^{\prime} \times j$ : $S^{1} \times I_{h} \rightarrow \mathbf{R}^{2} \times \mathbf{R}=\mathbf{R}^{3}$ such that $\gamma \times j$ is isometrically homotopic to $\gamma^{\prime} \times j$. If $h \geqslant 1$, then the tangent winding numbers of $\gamma$ and $\gamma^{\prime}$ are the same; if $h<1$, then the tangent winding numbers of $\gamma$ and $\gamma^{\prime}$ are the same modulo 2 .

We also have the following corollary.

8.7. Corollary. If $h \geqslant 1$, then $i: C_{h} \rightarrow \mathbf{R}^{3}$ and $r: C_{h} \rightarrow \mathbf{R}^{3}$ are not isometrically homotopic.

Proof. The isometric immersion $i$ is of the form $\gamma \times j$ where $\gamma: S^{1} \rightarrow \mathbf{R}^{3}$ is the inclusion map. The isometric immersion $r$ is given by $r(x, y, z)=(x, y$, $-z)$. By rotating about the $x$-axis we see that $r$ is isometrically homotopic to $\bar{r}: C_{h} \rightarrow \mathbf{R}^{3}$ where $\bar{r}(x, y, z)=(x,-y, z)$. The isometry $\bar{r}$ is of the form $\gamma^{\prime} \times j$ where $\gamma^{\prime}: S^{1} \rightarrow \mathbf{R}^{3}$ is given by $\gamma^{\prime}(x, y)=(x,-y)$. Since the tangent winding numbers of $\gamma$ and $\gamma^{\prime}$ are 1 and -1 respectively, the corollary follows from Proposition 8.6. Q.E.D. 
9. Unit of pseudofolding. In this section and the next we will construct a homotopy from $i$ to $r$ through isometric embeddings for $h<\pi /(\pi+2)$ and through isometric immersions for $h<1$.

The key to our method of inverting the cylinder $C_{h}$ is a way of nearly folding part of $C_{h}$ back and forth with the effect that a certain twisting is accomplished. We break this folding process into fundamental units. A detailed description of one of these fundamental units of near folding is given in this section.

We are going to show how to take a strip of width $2 h$ and length $l$ and homotopy it from a flat position to the one pictured in Figure 9.1. The salient facts concerning this homotopy are contained in Lemma 9.1.

Figure 9.1 is the inspiration for Lemma 9.1. The idea is to alter the crease lines so that they do not meet on the strip and then, not to actually fold at these lines but rather to make smooth bends in small neighborhoods of these lines. These bends will be called pseudofolds and the lines around which they are made are called pseudocrease lines.
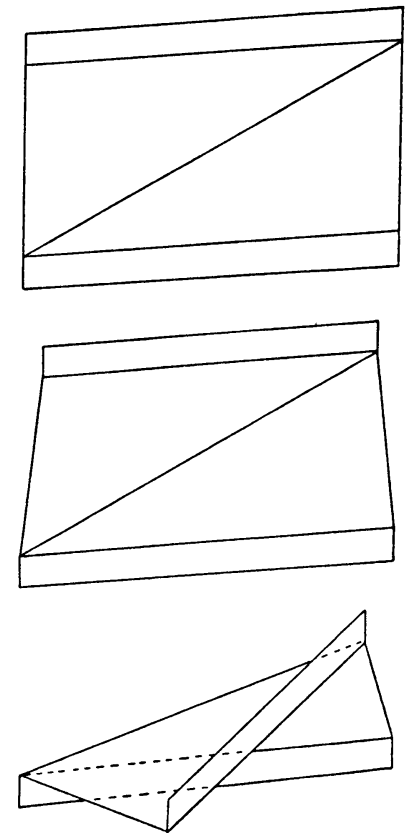

FIGURE 9.1

9.1. Lemma. For each $l>0$ there exists an $\varepsilon=\varepsilon(l)>0$ and $a \theta=\theta(l)$, and a smooth isotopy $\psi:[0, l] \times[-h, h] \times[0, \theta] \rightarrow \mathbf{R}^{3}$ through isometric embeddings such that with the notation $\psi_{t}(x, y)=\psi(x, y, t)$ :

1. $\psi_{0}(x, y)=(x, y, 0)$. (The strip starts in a flat position.)

2. $\psi_{t}(x, y)=(x, y, 0)$ for $x \leqslant \varepsilon$. (A front margin is held fixed.) 
3. $\psi_{t}(x, y)=T_{t} \circ R_{t}(x, y)$ for $x \geqslant l-\varepsilon$ where $T_{t}$ is a translation depending smoothly on $t$, and $R_{t}$ is the rotation about the $x$-axis by an angle of $t$ radius. (The back margin undergoes a twisting about the original lengthwise direction of the strip and a translation.)

4. $\psi_{t}(x, y)=(a, b, c)$ with $\varepsilon \leqslant a \leqslant P_{1} \circ \psi_{t}(l-\varepsilon, 0)$ for $\varepsilon \leqslant x \leqslant l-\varepsilon$, where $P_{1}: \mathbf{R}^{3} \rightarrow \mathbf{R}$ is the projection onto the first factor, $P_{1}(u, v, w)=u$. (The portion between the two end margins stays between them horizontally.)

5. $\psi_{t}(x, y)=(a, b, c)$ with $\left(b^{2}+c^{2}\right)^{1 / 2} \leqslant h+l e(l)$ for all $(x, y, t) \in[0$, $l] \times[-h, h] \times[0, \theta]$, where $e(l) \rightarrow 0$ as $l \rightarrow 0$. (The whole motion stays within a cylinder slightly larger than needed to contain the starting position.)

6. $\theta(l)=(1+\Delta(l)) l / h$ where $\Delta(l) \rightarrow 0$ as $l \rightarrow 0$. (The amount of twisting accomplished is $l / h$ to the first order.)

The truth of Lemma 9.1 is strongly suggested by the figure and discussion above. But since we are insisting on a smooth homotopy through isometric embeddings, some details of the proof of Lemma 9.1 seem appropriate.

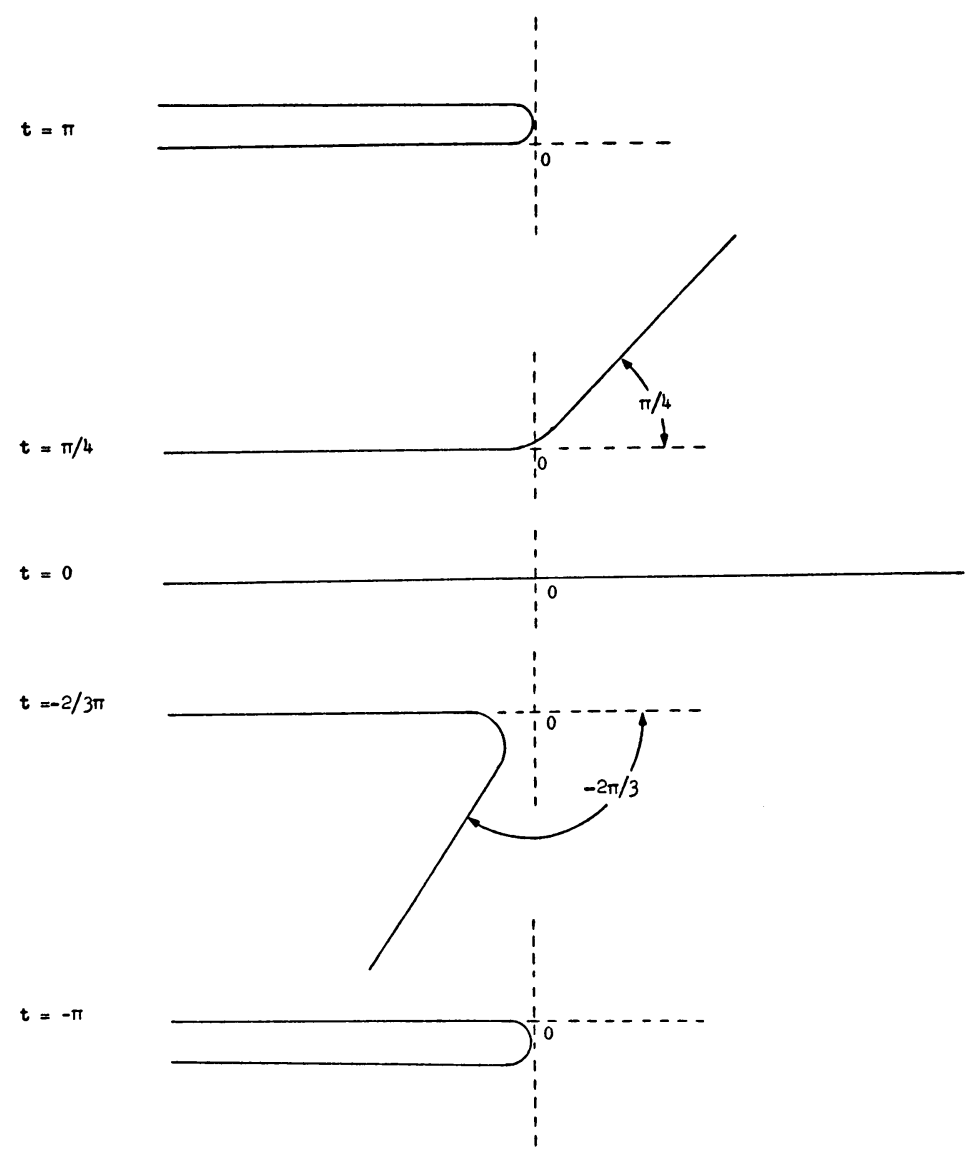

FIGURE 9.2 
Proof. First we show how to make one near fold which we call a pseudofold. Then we put three pseudofolds together to form the homotopy of Lemma 9.1. We base our pseudofold on an isometric isotopy of a curve $\gamma_{t}$ : $\mathbf{R} \rightarrow \mathbf{R}^{2}, t \in[-\pi, \pi]$.

This is pictured in Figure 9.2. One approach to defining $\gamma_{t}$ is to require it to be parameterized by arc length, coincide for a while with the negative $x$-axis as shown, and have its curvature $\kappa_{t}(s)$ given by $\kappa_{t}(s)=t \varphi(t)$ where $\varphi: \mathbf{R} \rightarrow \mathbf{R}$ is a smooth nonnegative bump function such that $\int \varphi(s) d s=1$. We leave the details to the reader.

By crossing $\gamma_{t}$ with $\mathbf{R}$ we obtain a pseudofold $\bar{\gamma}_{t}: \mathbf{R}^{2} \rightarrow \mathbf{R}^{3}, \bar{\gamma}_{t}(x, z)=\left(\gamma_{t}(x)\right.$, $z) \in \mathbf{R}^{2} \times \mathbf{R}^{1}=\mathbf{R}^{3}$. The line $x=0$ is the pseudocrease line, and $t$ is called the angle of pseudofolding. The length of the small portion of $\mathbf{R}$ where the curvature of $\gamma_{t}$ does not vanish is called the fudge factor because it is a measure of the difference between the pseudofold $\bar{\gamma}_{t}$ and an actual fold on the line $x=0$. We also let the term pseudofold refer to compositions of $\bar{\gamma}$ with isometries of $\mathbf{R}^{2}$ and of $\mathbf{R}^{3}$, and arbitrary smooth reparameterizations of the homotopy parameter $t$, and restrictions of these to subsets of $\mathbf{R}^{2}$.

We now describe the map $\psi_{t}$.

The space $[0, l] \times I_{h}$ is referred to as the strip and is denoted by $S$. The original or starting position of $[0, l] \times I_{h}$ is the inclusion map $[0, l] \times I_{h} \subset \mathbf{R}$ $\times \mathbf{R}=\mathbf{R} \times \mathbf{R} \times 0 \subset \mathbf{R}^{3}$. In the $u v$-plane angles are measured from positive $u$-axis to the positive $v$-axis. This applies to $(u, v)=(x, y),(x, z)$ and $(y, z)$. In Figure 9.3 the dashed lines $l_{1}, l_{2}$ and $l_{3}$ are potential pseudecrease lines. The regions $A, C, E$, and $G$ will always be mapped rigidly. All the curvature will be contained in the regions $B, D$ and $F$, and will be the result of making pseudofolds at $l_{1}, l_{2}$, and $l_{3}$. The points $X$ and $Y$ are the end points of the line segment $l_{2}$. The distance from $X$ to the left edge and the distance from $Y$ to

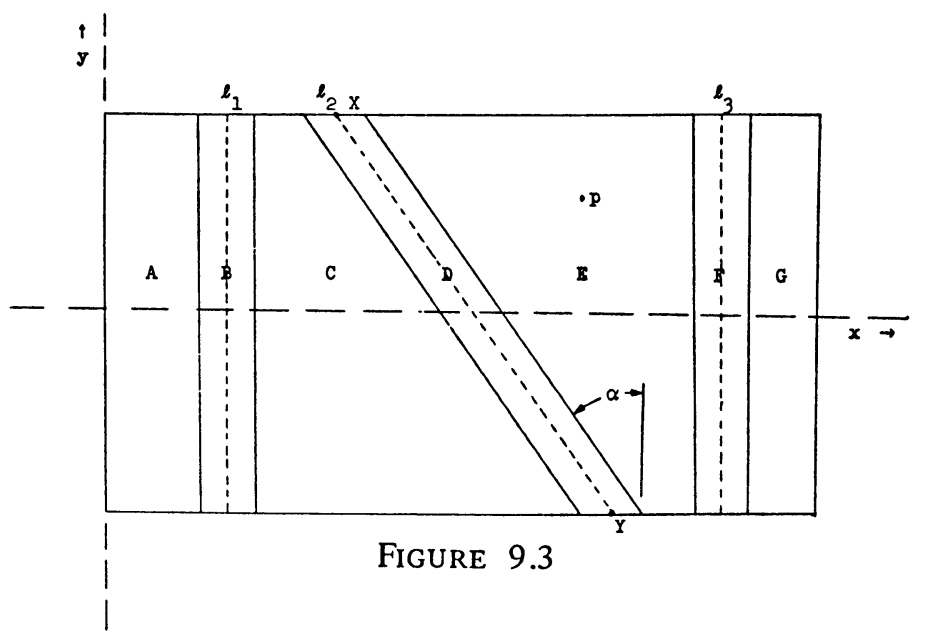


the right edge are required to be of the order of $\delta$ where $\delta$ is the fudge factor to be used in the pseudofolds. Let $\delta$ be fixed for now.

If we specify the angles of pseudofold at each pseudocrease line along with the position of the first rigid section, section $A$, a unique isometric immersion from $S$ into $\mathbf{R}^{3}$ is determined. We restrict ourselves to the cases where section $A$ is specified to be in its original position. In this way we get a function $\varphi$ : $(-\pi, \pi)^{3} \times S \rightarrow \mathbf{R}^{3}$ so that $x \rightarrow \varphi\left(\theta_{1}, \theta_{2}, \theta_{3}, x\right)$ is the isometric immersion determined by the angles of pseudofolding $\theta_{1}, \theta_{2}$ and $\theta_{3}$. The function $\varphi$ is clearly smooth. What we must do is describe how to find $\theta_{1}, \theta_{2}$ and $\theta_{3}$ as smooth functions of the homotopy parameter $t$ so that conditions $1-6$ of Lemma 9.1 are satisfied by

$$
\psi(x, t)=\varphi\left(\theta_{1}(t), \theta_{2}(t), \theta_{3}(t), x\right), \quad(x, t) \in S \times[0, \theta(l)] .
$$

Given a $t \in[0, \pi / 2-\delta]$, the angles $\theta_{1}(t)$ and $\theta_{3}(t)$ are easy. We simply set $\theta_{1}(t)=\theta_{3}(t)=t$. In order to find the correct value for $\theta_{2}(t)$ we proceed as follows. We divide the strip into regions or sections as indicated in Figure 9.3. Let $U=A \cup B \cup C \cup D \cup E$ and $V=D \cup E \cup F \cup G$. We will construct isometric embeddings $f: U \rightarrow \mathbf{R}^{3}$ and $g: V \rightarrow \mathbf{R}^{3}$ by making pseudofolds at $l_{1}, l_{2}$ and $l_{3}$. We will arrange things so that $f$ and $g$ agree on $E$, and the resulting map $\psi_{t}^{\circ}: U \cup V=S \rightarrow \mathbf{R}^{3},\left(\psi_{t}^{\circ} \mid U=f\right.$ and $\psi_{t}^{\circ}|E \cup F \cup G=g| E$ $\cup F \cup G$ ) has the desired properties. It will be manifestly apparent from our constructions of $f$ and $g$ that both map $E$ rigidly. Hence, to show that they agree on $E$ it will be sufficient to show that they agree and their unit normal vectors agree at a single point $p \in E$, and their differentials at $p$ agree on the tangent vector $Y-X$. We use this observation to guide our construction.

Let $f^{1}: U \rightarrow \mathbf{R}^{3}$ be determined by requiring that $A$ is held fixed in its original position and there is a pseudofold at $l_{1}$ of angle $t$. Similarly, $g^{1}$ : $V \rightarrow \mathbf{R}^{3}$ is defined by requiring that $G$ is held fixed in its original position and that there is a pseudofold at $l_{3}$ of angle $t$. Consider the vectors determined by the new positions of the directed line segment $X Y$, i.e., consider $l^{1}=f^{1}(Y)-$ $f^{1}(X)$ and $\bar{l}^{1}=g^{1}(Y)-g^{1}(X)$. Let $P_{y z}: \mathbf{R}^{3} \rightarrow \mathbf{R}^{2}$ be the projection onto the $y z$-plane, $P_{y z}(a, b, c)=(b, c)$. Let $w(t)$ be the angle from $P_{y z} l^{1}$ to $P_{y z} l^{1}$. Then $0 \leqslant w(t) \leqslant 2 \alpha$ (see Figure 9.3 for $\alpha$ ). We require $l$ to be sufficiently small so that $2 \alpha<\pi$.

Compose $g^{1}$ with $R=$ the rotation about the $x$-axis by an angle of $-w(t)$, and obtain $g^{2}=R \circ g^{1}: V \rightarrow \mathbf{R}^{3}$. Now look at $\bar{l}^{2}=g^{2}(Y)-g^{2}(X)$. Clearly $P_{y z} \bar{l}^{2} \| P_{y z} l^{1}$. Next, consider the $x$-components of $l^{1}, \bar{l}^{1}$ and $\bar{l}^{2}$. They are all equal because $f^{1}$ and $g^{1}$ rotate $X Y$ about lines parallel to the $y$-axis by angles $t$ and $-t$ respectively, and $R$ does not change $x$-components. Letting $P_{x}$ : $\mathbf{R}^{3} \rightarrow \mathbf{R}$ be the projection on the $x$-axis, $P_{x}(a, b, c)=a$, we have $P_{x} \bar{l}^{2}=P_{x} l^{1}$. We also know that $\left\|\bar{l}^{2}\right\|=\left\|l^{1}\right\|$ because $g^{2}$ and $f^{1}$ act rigidly on section $D$. It follows that $\bar{l}^{2}=l^{1}$. 
Let $n^{1}, \bar{n}^{1}$ and $\bar{n}^{2}$ be the unit normal vectors to $f^{1}, g^{1}$ and $g^{2}$ at the fixed point $p \in E$. Let $\theta_{2}(t)$ be the angle from $n^{1}$ to $\bar{n}^{2}$ measured in the positively oriented coordinate system $x^{\prime}, y^{\prime}, z^{\prime}$ in which $l^{1}=l^{2}$ points along the positive $x^{\prime}$-axis. We will show that $\theta_{2}(t)$ is well defined and satisfies $\left|\theta_{2}(t)\right|<\pi$. It is sufficient to show that $n^{1} \neq-\bar{n}^{2}$. Recall that $2 \alpha<\pi$. Since $0 \leqslant t<\pi / 2$, $P_{y z} n^{1}=P_{y z} \bar{n}^{-1} \neq 0$. Hence, the angle between $P_{y z} n^{1}$ and $P_{y z} \bar{n}^{2}$ is $-w(t)$. But $-\pi<-2 \alpha \leqslant-w(t) \leqslant 0$. Hence, $P_{y z} n^{1} \neq-P_{y z} \bar{n}^{2}$, and therefore $n^{1} \neq$ $-\bar{n}^{2}$. Now it is easy to supply an appropriate formula for $\theta_{2}(t)$ which shows that $\theta_{2}(t)$ is a smooth function of $t$.

We continue to construct the promised functions $f$ and $g$ in order to show that $\theta_{2}(t)$ has the desired properties. Let $f^{2}: U \rightarrow \mathbf{R}^{3}$ be determined by pseudofolds at $l_{1}$ and $l_{2}$ of angles $\theta_{1}(t)=t$ and $\theta_{2}(t)$ respectively. Let $l_{2}$ be the new position of $X Y, l^{2}=f^{2}(Y)-f^{2}(X)$. The line segment $X Y$ is just translated in the transition from $f^{1}$ to $f^{2}$ and therefore $l^{2}=l^{1}=l^{2}$. Note that $n^{1}$ and $\bar{n}^{2}$ are perpendicular to $l^{1}=\vec{l}^{2}$. Also, the transition from $n^{1}$ to $n^{2}=$ the normal to $f^{2}$ at $p$, is simply a rotation about the direction of $l^{1}$, i.e., the $x^{\prime}$-axis, by the angle $\theta_{2}(t)$. Hence $n^{2}=\bar{n}^{2}$. Now let $T: \mathbf{R}^{3} \rightarrow \mathbf{R}^{3}$ be the translation which takes $g^{2}(p)$ to $f^{2}(p)$. Set $f=f^{2}$ and $g=T \circ g^{2}$. The maps $f$ and $g$ agree on $E$ and so $f$ and $g \mid E \cup F \cup G$ combine to give the desired function $\psi_{i}^{\circ}: S \rightarrow \mathbf{R}^{3}$. To obtain property 3 we reparameterize the homotopy

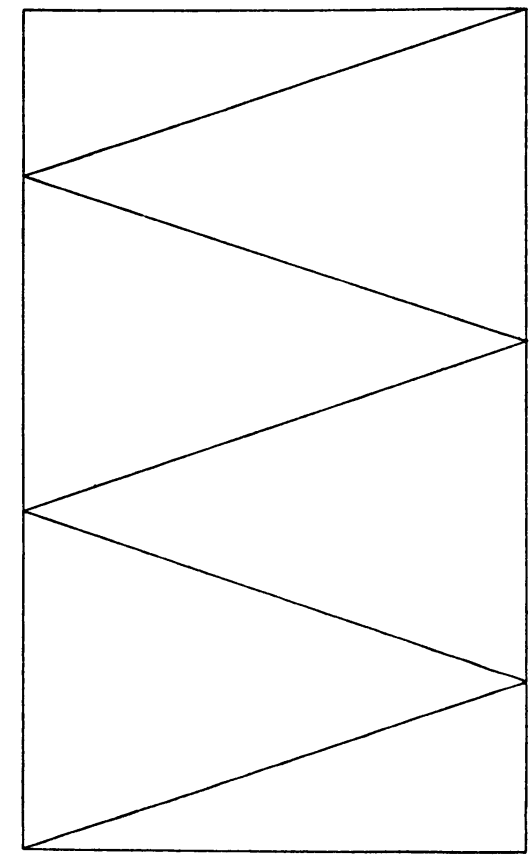

FIGURE 9.4 BEFORE

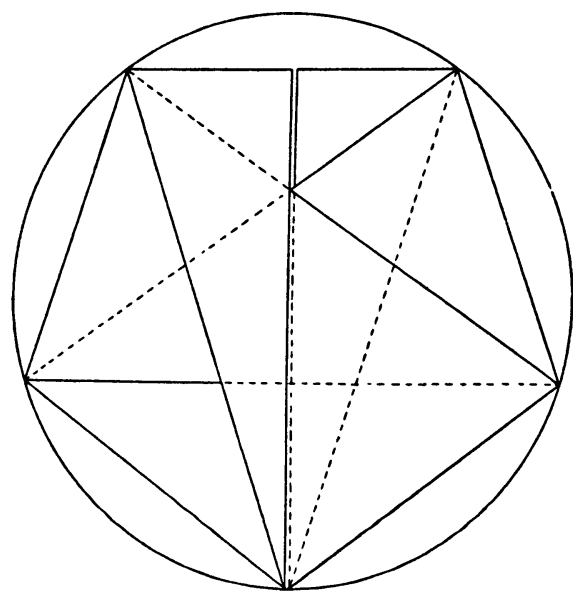

FIGURE 9.4 AFTER 
by $w^{-1}:[0, \theta] \rightarrow[0, \pi / 2-\delta]$ where $\theta=\theta(l)=w(\pi / 2-\delta)$; we replace $\psi_{i}^{\circ}$ by $\psi_{t}=\psi_{w^{-1}(t)}^{0}$. By making $\delta$ small the smooth homotopy $\psi_{t}$ approaches the folded one indicated in Figure 9.1. Properties 5 and 6 of Lemma 9.1 follow from this. Properties 1, 2, and 4 clearly hold from the construction. Q.E.D.

REMARK. The purpose of property 5 in Lemma 9.1 with its careful estimate "le $(l)$ " is to enable one to make several elementary units of pseudofolding one after the other and still have the whole motion stay within a cylinder slightly larger than needed to contain the starting position. This also can be seen by noting that as the fudge factors approach zero a sequence of units of pseudofoldings approach actual foldings which have this property. See Figure 9.4.

10. The inversion. Naturally enough, we refer to any isometric homotopy between $i: C_{h} \rightarrow \mathbf{R}^{3}$ and $r: C_{h} \rightarrow \mathbf{R}^{3}$ as an inversion. We will use several diagrams to describe a particular inversion $H_{t}$. These diagrams are abstractions of overhead views, i.e., projections onto the $x y$-plane of a sequence of stages of the inversion. First we will explain the significance of various components of these diagrams. A smooth line represents the image of a portion of the cylinder for which $H_{t}$ can be written as $\gamma \times j: U \times I_{h} \rightarrow \mathbf{R}^{2} \times$ $\mathbf{R}=\mathbf{R}^{3}$, where $\gamma: U \rightarrow \mathbf{R}^{2}$ is a smooth curve defined on a subset $U$ of $S^{1}$ and $j: I_{h} \rightarrow \mathbf{R}$ is the inclusion map. When $\gamma$ folds $U$ back and forth many times so that $\gamma \times j$ makes $U \times I_{h}$ look like an accordion, we denote this by

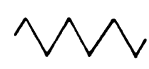

and refer to this portion as an accordion. When an accordion is rotated about the $x$-axis we renresent it by something like

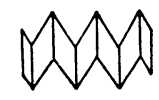

A fundamental unit of pseudofolding (as described in Lemma 9.1) in progress is denoted by

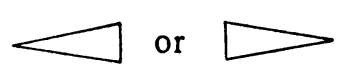

Accumulations of several completed units are denoted by

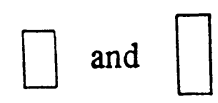




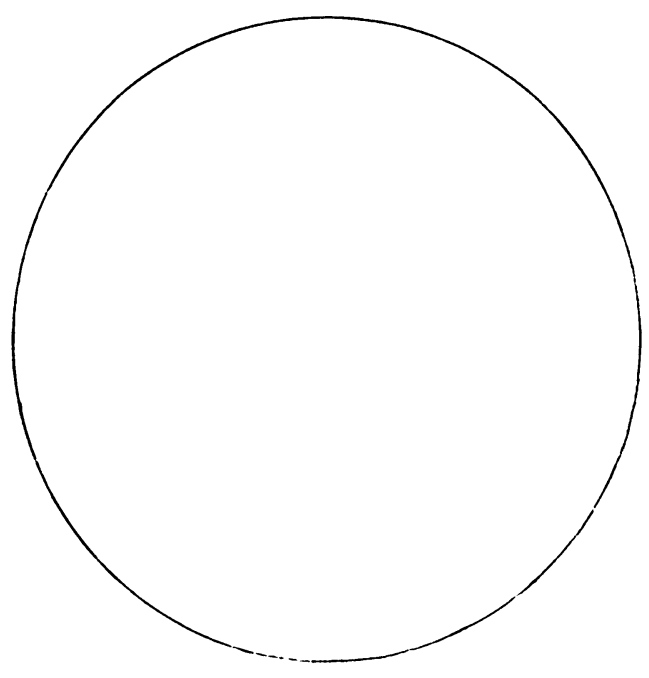

Figure 10.1a

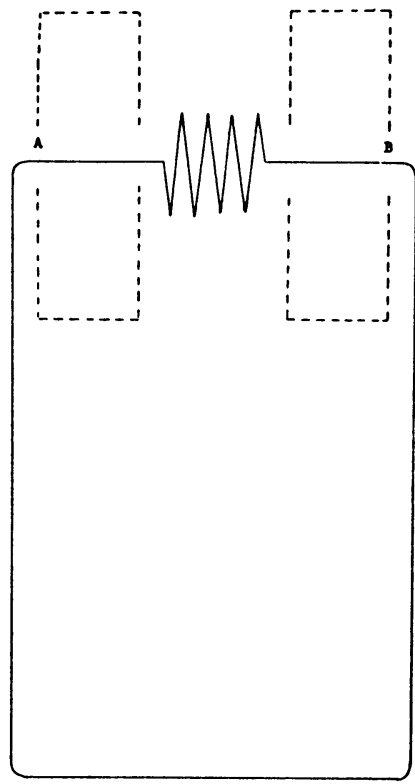

FIGURE $10.1 \mathrm{~b}$

Figure 10.1a represents the starting position $H_{0}=i$. In Figure $10.1 \mathrm{~b}, H_{t}$ is of the form $\gamma \times j: S^{1} \times I_{h} \rightarrow \mathbf{R}^{2} \times \mathbf{R}=\mathbf{R}^{3}$, and there is an accordion at the top. The dashed rectangles are not part of $H_{t}$. They are to be thought of as pseudofolding machines. The cylinder will be referred to as the tape in order to suggest its flexibility. The tape will now start to be fed into the pseudofolding machines at $A$ and $B$. One fundamental unit of pseudofolding is done at a time in each machine simultaneously. The completed units are accumulated at the sides of the accordion and the accordion rotates in one direction. This is indicated in Figures 10.1c and 10.1d. In Figure 10.1e the accordion has rotated through $180^{\circ}$ and much of the tape has accumulated in two banks of completed units of pseudofolding. Figure 10.1f shows these banks being rotated away from each other while the accordion expands. Figure $10.1 \mathrm{~g}$ is the midway position of the inversion. In Figure $10.1 \mathrm{~h}$ the banks continue to rotate and an accordion is beginning to form on the other side. The rest of the inversion is analogous to the first half.

The above can be used as a description of a homotopy through isometric embeddings from $i: C_{h} \rightarrow \mathbf{R}^{3}$ to $r: C_{h} \rightarrow \mathbf{R}^{3}$ provided there is enough length in the circumference of $S^{1}$ (in relation to $h$ ) in order to accomodate the two accordions and the two banks of units of pseudofolding. This will be the case if $2 \pi>2 h+2 h+\pi h+\pi h$. Hence, if $h<\pi /(\pi+2)$, then $i$ is isometrically isotopic to $r$. 


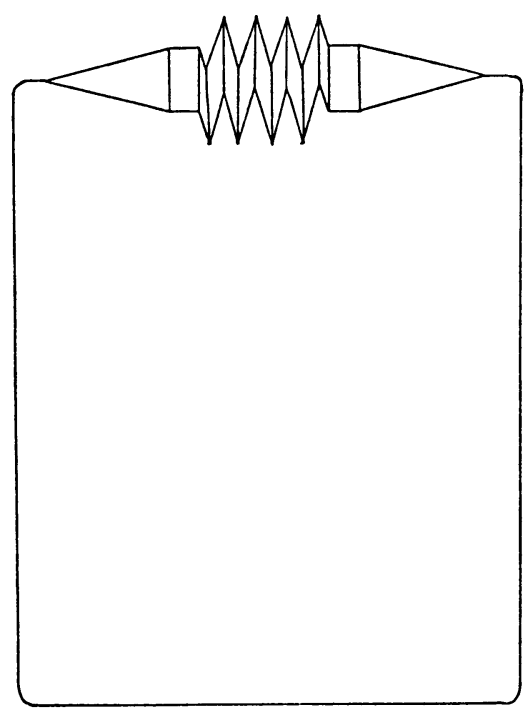

FiguRE 10.1c

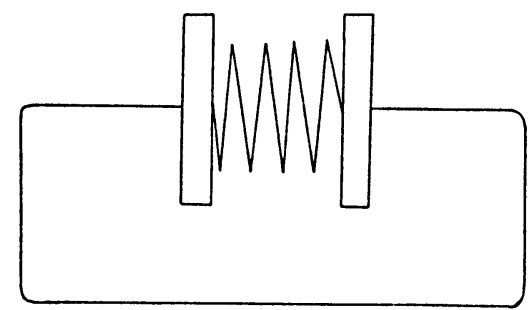

FIGURE 10.1e

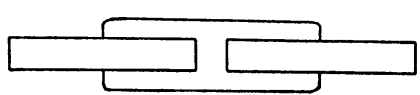

F IGURE $10.1 \mathrm{~g}$

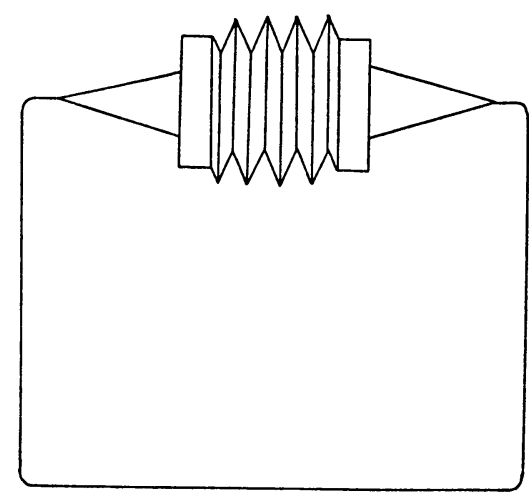

FiguRE 10.1d

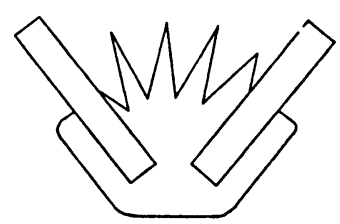

FIGURE $10.1 \mathrm{f}$

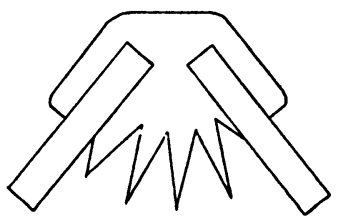

FIGURE $10.1 \mathrm{~h}$

If we wish to use the above as a description of a homotopy from $i$ to $r$ through isometric immersions, we do not have to worry about the accordions. In the stages corresponding to Figures $10.1 \mathrm{f}, 10.1 \mathrm{~g}$ and $10.1 \mathrm{~h}$, the banks may be passed through each other. Hence, it is sufficient for $2 \pi>\pi h+\pi h$. Therefore, if $h<1$, then $i$ is isometrically homotopic to $r$. Summing up we have proved the following theorems. 
10.2. THEOREM. The inclusion $i$ is isometrically homotopic to $r$ if and only if $h<1$.

10.3. THEOREM. The inclusion $i$ is isometrically isotopic to $r$ if

$$
h<\pi /(\pi+2) \text {. }
$$

An amusing paper model which demonstrates that $i$ is isometrically homotopic to $r$ for $h<\pi / 6$ (note $\pi / 6 \sim 0.52$ where as $\pi /(\pi+2) \sim 0.61$ ) can be made from a strip of paper with fold lines as indicated in Figure 10.2, and with $A^{\prime}$ pasted on $A$. It is not completely trivial to invert this model using only the indicated fold lines and as a hint we offer in Figure 10.3 an illustration of the middle position in the inversion.

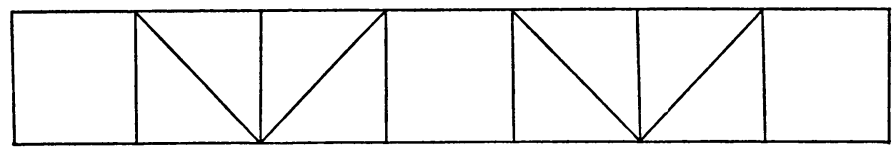

FIGURE 10.2

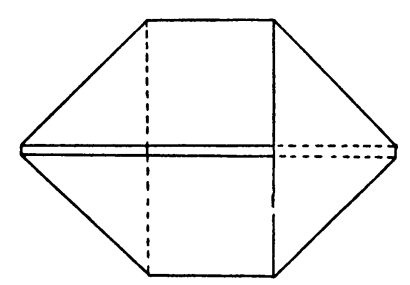

FIGURE 10.3

11. Extensions to isometric immersions of the form $\gamma \times j$. In this section we prove the following theorem.

11.1. THEOREM. Given two isometric immersions of the form

$$
\gamma \times j, \gamma^{\prime} \times j: S^{1} \times I_{h} \rightarrow \mathbf{R}^{2} \times \mathbf{R}^{1}=\mathbf{R}^{3},
$$

if $h \geqslant 1(h<1)$, then $\gamma \times j$ and $\gamma^{\prime} \times j$ are isometrically homotopic iff the tangent winding numbers of $\gamma$ and $\gamma^{\prime}$ are equal (equal modulo 2).

ProOF. The "only if" direction of the implication has already been established in Proposition 8.6. The "if" direction will be proved using the following two observations. Let $\mathscr{W}(\gamma)$ denote the tangent winding number of $\gamma$ : $S^{1} \rightarrow \mathbf{R}^{2}$.

(1) If $\mathcal{W}\left(\gamma_{0}\right)=W\left(\gamma_{1}\right)$, then $\gamma_{0} \times j$ is isometrically homotopic to $\gamma_{1} \times j$.

(2) If $h<1$, then for each isometric immersion of the form $\gamma \times j$ there are 
isometric immersions of the forms $\varphi \times j$ and $\psi \times j$ such that $\mathcal{W}(\varphi)=\mathscr{\vartheta}(\gamma)$ +2 and $W(\psi)=W(\gamma)-2$.

The case $h \geqslant 1$ follows immediately from (1). The case $h<1$ follows easily from (1) and repeated use of (2).

To establish (1) we recall that the Whitney-Graustein Theorem [10] asserts that two closed curves in $\mathbf{R}^{2}$ are regularly homotopic iff their tangent winding numbers are equal. Given two isometric immersions $\gamma_{0}, \gamma_{1}: S^{1} \rightarrow \mathbf{R}^{2}$, we know by Whitney-Graustein that there is a regular homotopy $\gamma_{t}: S^{1} \rightarrow \mathbf{R}^{2}, 0 \leqslant t \leqslant$ 1 , between $\gamma_{0}$ and $\gamma_{t}$. The intermediate curves $\gamma_{t}$ may not be isometric. So let $L(t)$ be the total arc length of $\gamma_{t}$. Set $\bar{\gamma}_{t}(s)=(2 \pi / L(t)) \gamma_{t}(s)$. Now reparameterize each $\bar{\gamma}_{t}$ by arc length measured counterclockwise from a fixed point on $S^{1}$. The resulting family of curves $\gamma_{t}^{*}$ is an isometric homotopy from $\gamma_{0}$ to $\gamma_{1}$. It follows that $\gamma_{t}^{*} \times j$ is an isometric homotopy from $\gamma_{0} \times j$ to $\gamma_{1} \times j$. This establishes (1).

To establish (2) we first note that given $\gamma$ there exists an isometric immersion $\gamma^{\prime}: S^{1} \rightarrow \mathbf{R}^{2}$ such that

(a) $\mathscr{W}\left(\gamma^{\prime}\right)=\mathscr{W}(\gamma)$

(b) $\gamma^{\prime}$ restricted to $\{\bar{\pi}(s) \mid 0 \leqslant s \leqslant 2 \pi-\varepsilon\}$ traces out a circle counterclockwise which is tangent to the $x$-axis at $s=0$, and lies in the upper half-plane. The positive number $\varepsilon$ satisfies $h<(2 \pi-\varepsilon) / 2 \pi$. (Recall $\bar{\pi}(s)=(\cos s$, sin s).) This is obvious and we leave the details of the construction to the interested reader.

By (1) and (a), $\gamma \times j$ is isometrically homotopic to $\gamma^{\prime} \times j$. Now the inversion described in $\S \S 9$ and 10 can be used to produce an isometric homotopy from $\gamma^{\prime} \times j$ to an isometry of the form $\psi \times j$ where $\psi$ agrees with $\gamma^{\prime}$ on $\{\bar{\pi}(s) \mid 2 \pi-\varepsilon \leqslant s \leqslant 2 \pi\}$; and on $\{\bar{\pi}(s) \mid 0 \leqslant s \leqslant 2 \pi-\varepsilon\}, \psi=R \circ \gamma^{\prime}$ with $R$ the reflection in the $x$-axis, $R(x, y)=(x,-y)$. During this isometric homotopy the set $\{\bar{\pi}(s) \mid 2 \pi-\varepsilon \leqslant s \leqslant 2 \pi\}$ is held fixed. Clearly $\mathcal{W}(\varphi)=$ W $\left(\gamma^{\prime}\right)-2=\mho(\gamma)-2$. Similarly, we can construct an isometric immersion $\varphi \times j$ isometrically homotopic to $\gamma \times j$ and such that $\mathscr{W}(\varphi)=\mathscr{W}(\gamma)+2$. Q.E.D.

12. Immersing the Möbius strip. Using pseudofolds we will describe how to isometrically immerse $M_{l}$ into $\mathbf{R}^{3}$ for any $l>\pi / 2$. Combined with Corollary 5.2 , this completely answers the question as to when a Möbius strip may be isometrically immersed in $\mathbf{R}^{3}$.

12.1. THEOREM. A Möbius strip isometrically immerses in $\mathbf{R}^{3}$ iff the ratio of its length to its width is greater than $\pi / 2$. That is, $M_{l}$ immerses in $\mathbf{R}^{3}$ iff $l>\pi / 2$.

Proof. We will describe how to isometrically immerse $M_{l}$ with $l>\pi / 2$ into $\mathbf{R}^{3}$ in terms of what to do with a strip of paper. The strip is divided into three rectangular sections and pseudocrease lines are drawn as in Figure 12.1. 
The pseudocrease lines are the dashed lines. If $n$ is the number of pseudocrease lines inside the rectangle $A B C D(n=5$ in Figure 12.1) and $w$ is the positive angle each makes with the vertical direction, then $n$ is odd and $n 2 w=\pi$. By pseudofolding back and forth alternately along the pseudocrease lines in the first section (rectangle $A B C D$ ) the directed line segment $\overrightarrow{C D}$ may be brought to a position antiparallel to $\overrightarrow{A B}$. This construction is similar to the one pictured in Figure 9.4. Consider Figure 12.1. The gaps are the small distances between the appropriate ends of successive pseudocrease lines. Under the above pseudofolding, the point $D$ will end up near $A$ provided the gaps and the fudge factors are sufficiently small. This is because as the gaps and fudge factors go to zero, the rectangle $A B C D$ "pseudofolded up" as described above approaches a rectangle $A B C D$ folded up using actual folds along the lines indicated in Figure 12.1. With actual folds and no gaps $C D$ lands on $A B$.

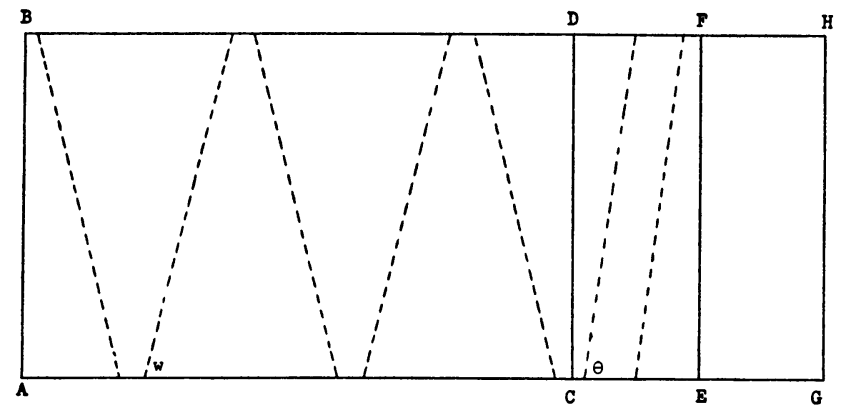

FIGURE 12.1

Now using the second portion of paper (rectangle $C D F E$ ) with two parallel pseudocrease lines as shown in Figure 12.1, we can adjust $\theta$ so that by pseudofolding back and forth by the same amount on these two crease lines, the segment $E F$ will be brought to a position which is the position $B A$ translated in a direction perpendicular to $B A$. Finally, using the third rectangle of paper $E F H G$ we can easily bring $G H$ to coincide with $B A$ so that the two ends of the strip join smoothly. By making the number of pseudofolds in rectangle $A B C D$ sufficiently large and the gaps and fudge factors sufficiently small, the distance $\overline{A C}$ can be made arbitrarily close to $\pi / 2$ (see Figure 9.4). Similarly the distance from the pseudofolded-up position from $D$ to $A$ can be made arbitrarily small and hence the distances $\overline{C E}$ and $\overline{E G}$ can be chosen arbitrarily small. Q.E.D.

13. For $h$ near 1 any inversion is like our example. In this section we will show that if $g$ is an inversion of the cylinder $C_{h}$ with $h$ very close to 1 , then $g$ must resemble the inversion described in $\S 10$. It will be seen that for some 
intermediate value $\tau_{0}$ of the homotopy parameter $\tau$, the immersion $g_{\tau_{0}}$ must "nearly fold" the cylinder many times. To simplify some of our statements we sometimes drop the ever present phrase "for $h$ sufficiently close to $1 . "$

Suppose $9: C_{h} \times I \rightarrow \mathbf{R}^{3}$ is an inversion of the cylinder $C_{h}$, that is, $g$ is an isometric homotopy from $i$ to $r$. By the development leading up to Corollary 8.7 we see that there must be a $\tau$ such that $g_{\tau}: C_{h} \rightarrow \mathbf{R}^{3}$ has a pair of antiparallel generating lines. Suppose these occur at $s=\alpha$ and $s=\beta$.

Set $f=\mathscr{g}_{\tau}$ and $g=f \circ \pi: \mathbf{R} \times I_{h} \rightarrow \mathbf{R}^{3}$. We may parameterize the generating lines of $g$ as in $\$ 5$ and we assume all the notation from the proof of Proposition 5.1. In order to simplify the following discussion we will assume $T$ and $B$ are continuously differentiable. This assumption is not necessary and can be removed as it was removed in $\S 5$.

Recall that the curve $\varphi(s), s \in[\alpha, \beta]$, goes from a point on one central ray to a point on the opposite central ray while all the time staying outside the open ball of radius $2 h$, i.e., $\|\varphi(s)\| \geqslant 2 h$ for all $s \in[\alpha, \beta]$. Since $g$ is periodic of period $2 \pi$ we may choose $\alpha$ and $\beta$ such that $\alpha<\beta$ and $\beta-\alpha \leqslant \pi$. It was shown in $\S 5$ that the length of $\varphi:[\alpha, \beta] \rightarrow \mathbf{R}^{3}$ is no more than $2(\beta-\alpha)$. Obviously the length $\varphi$ is no less than $2 \pi h$. Hence, for $h$ sufficiently close to 1 we may conclude that the curve $\varphi$ is rather tightly bound about the ball of radius $2 h$. That is, we conclude that for all $s \in[\alpha, \beta]$ :

$$
\|\varphi(s)\| \text { is arbitrarily close to } 2 h,
$$

$\varphi(s)$ is arbitrarily near the plane $P$ determined by the three points $\varphi(\alpha)$, $\varphi((\alpha+\beta) / 2)$ and $\varphi(\beta)$.

Also

for all $s$ outside a set of arbitrarily small measure, $\varphi^{\prime}(s)$ is nearly perpendicular to $\varphi(s)$ and nearly lies in $P$ to a fixed side of $\varphi(s)$.

These facts are intuitively obvious and follow readily using spherical coordinates.

Next, we will see what (1), (2) and (3) imply about $T$ and $B$. It follows from (1) that

$$
\begin{aligned}
& \sup _{\alpha \leqslant s<\beta}|T(s)-B(s)| \text { can be made } \\
& \text { arbitrarily small by insisting that } h \text { is }
\end{aligned}
$$
sufficiently close to 1 .

It is an elementary theorem of differential geometry that on a flat surface the normal is constant along straight lines in the surface [6, p. 68]. Applying 
this to $g$ we see that the normal to $g$ is constant along the curve $\gamma$ obtained by restricting $g$ to the segment from $(B(s),-h)$ to $(T(s), h)$. Since $\gamma$ is a straight line parameterized by arc length its tangent is constant. Since $\left.d g\right|_{(s, t)}$ is an isometric map, we can conclude that the vector $\partial g /\left.\partial s\right|_{(s, t)}$ is constant along $\gamma$. Hence

$$
\begin{aligned}
\varphi^{\prime}(s) & =\left.\frac{\partial g}{\partial s}\right|_{(T(s), h)} T^{\prime}(s)-\left.\frac{\partial g}{\partial s}\right|_{(B(s),-h)} B^{\prime}(s) \\
& =\left.\frac{\partial g}{\partial s}\right|_{(s, 0)}\left(T^{\prime}(s)-B^{\prime}(s)\right) .
\end{aligned}
$$

From (5) we have $\left\|\varphi^{\prime}(s)\right\|=\left|T^{\prime}(s)-B^{\prime}(s)\right|$ and thus

$$
\int_{\alpha}^{\beta}\left|T^{\prime}(s)-B^{\prime}(s)\right| d t \geqslant 2 \pi h .
$$

Recall that $s=\frac{1}{2}(T(s)+B(s))$. Hence

$$
T^{\prime}(s)+B^{\prime}(s)=2 .
$$

Since $T$ and $B$ are monotone nondecreasing we have

$$
T^{\prime}(s) \geqslant 0 \text { and } B^{\prime}(s) \geqslant 0 .
$$

It follows from (7) and (8) that

$$
\left|T^{\prime}(s)-B^{\prime}(s)\right| \leqslant 2 .
$$

From (6) and (9) and $\beta-\alpha \leqslant \pi$ it follows that, for $h$ sufficiently close to 1 ,

$$
\left|T^{\prime}(s)-B^{\prime}(s)\right| \text { is nearly } 2 \text { for most } s \in[\alpha, \beta] \text {. }
$$

Note that when $T^{\prime}(s)-B^{\prime}(s)$ is near 2 we have because of (7) and (8) that $T^{\prime}(s)$ is near 2 and $B^{\prime}(s)$ is near 0 . Similarly, when $T^{\prime}(s)-B^{\prime}(s)$ is near -2 , we have $T^{\prime}(s)$ near 0 and $B^{\prime}(s)$ near 2.

From (4) we deduce

$$
\begin{aligned}
& \left|\int_{a}^{b}\left(T^{\prime}(s)-B^{\prime}(s)\right) d s\right| \text { is arbitrarily } \\
& \text { small uniformly for } a, b \in[\alpha, \beta] .
\end{aligned}
$$

Now, from (9), (10) and (11) we see that, for each $\varepsilon>0$, there exists a $\delta>0$ such that if $h \geqslant 1-\delta$ and $|b-a| \geqslant \varepsilon$, then

$$
\begin{aligned}
& m\left\{s \in[a, b] \mid T^{\prime}(s)-B^{\prime}(s) \geqslant 2-\varepsilon\right\} \geqslant \frac{1}{2}|b-a|-\varepsilon^{2} \text { and } \\
& m\left\{s \in[a, b] \mid T^{\prime}(s)-B^{\prime}(s) \leqslant-2-\varepsilon\right\} \geqslant \frac{1}{2}|b-a|-\varepsilon^{2}
\end{aligned}
$$

where $m A=$ the Lebesgue measure of $A$ for Lebesgue measurable sets $A \subset \mathbf{R}$. From (3) and (12) we see that we can find an arbitrarily long sequence $\alpha<s_{1}<s_{2}<\cdots<s_{N}<\beta$ such that

for $i$ even, $T^{\prime}\left(s_{i}\right)$ is near 2 and $B^{\prime}\left(s_{i}\right)$ is near 0 and for $i$ odd, $T^{\prime}\left(s_{i}\right)$ is near 0 and $B^{\prime}\left(s_{i}\right)$ is near 2 , 
and

for all $i, \varphi^{\prime}\left(s_{i}\right)$ is nearly perpendicular to $\varphi\left(s_{i}\right)$, and $\varphi^{\prime}\left(s_{i}\right)$ nearly lies in the plane $P$ to a fixed side of $\varphi\left(s_{i}\right)$,

and

$$
\begin{array}{cc}
s_{i+1}-s_{i}<2 \pi / N & \text { for } 1 \leqslant i \leqslant N-1, \\
s_{1}-\alpha<2 \pi / N, \quad & \beta-s_{N}<2 \pi / N .
\end{array}
$$

By taking $N$ large and using the following estimate which does not depend on $h$,

$$
\left\|\varphi(s)-\varphi\left(s^{\prime}\right)\right\| \leqslant 2\left|s-s^{\prime}\right|,
$$

we see that $\varphi^{\prime}\left(s_{i}\right)$ is nearly parallel to $\varphi^{\prime}\left(s_{i+1}\right)$, that is,

$$
\begin{aligned}
& \varphi^{\prime}\left(s_{i}\right) \text { is nearly equal to a positive } \\
& \text { multiple of } \varphi^{\prime}\left(s_{i+1}\right) \text {. }
\end{aligned}
$$

From (5) and (13) we see that $\partial g /\left.\partial s\right|_{\left(s_{i}, 0\right)}$ is near $\frac{1}{2} \varphi^{\prime}\left(s_{i}\right)$ for $i$ even, and near $-\frac{1}{2} \varphi^{\prime}\left(s_{i}\right)$ for $i$ odd. Hence, from (17) we have

$$
\partial g /\left.\partial s\right|_{\left(s_{i}, 0\right)} \text { is nearly equal to a positive }
$$

$$
\begin{aligned}
& \text { multiple of }-\partial g /\left.\partial s\right|_{\left(s_{i+1}, 0\right)} \text { for } 1 \leqslant i \leqslant \\
& N-1 \text {. }
\end{aligned}
$$

Since $\left\|\partial g /\left.\partial s\right|_{(s, 0)}\right\|=1$ for all $s$, we conclude that from (18) that the angle between $\partial g /\left.\partial s\right|_{\left(s_{i}, 0\right)}$ and $\partial g /\left.\partial s\right|_{\left(s_{i+1}, 0\right)}$ is nearly $\pi$. This and (15) shows that the curvature of the central curve $s \rightarrow g(s, 0)$ is rather large somewhere between $s_{i}$ and $s_{i+1}$. The normal to the surface at $\left(s_{i}, 0\right)$ is given by $\left(\partial g /\left.\partial s\right|_{\left(s_{i}, 0\right)} \times \varphi\left(s_{i}\right)\right)^{\wedge}$ where $V^{\wedge}=V /\|V\|$ for $V \in \mathbf{R}^{3}-\{0\}$. Thus from (17) and (18) we know that

the normal at $\left(s_{i}, 0\right)$ is nearly equal to minus the normal at $\left(s_{i+1}, 0\right)$, for $1 \leqslant i \leqslant N-1$.

We interpret (19) as saying that the surface is essentially folded over between $s_{i}$ and $s_{i+1}$.

From (5), (12) and the discussion following (10) we see that for any interval $[a, b] \subset[\alpha, \beta]$ with $|b-a| \geqslant \varepsilon$, there are subsets $A, B \subset[a, b]$ such that $m A$ is nearly $\frac{1}{2}|b-a|$, and $m B$ is nearly $\frac{1}{2}|b-a|$, and there is a constant vector $V$ such that $\partial g /\left.\partial s\right|_{(s, 0)}$ is nearly $V$ for $s \in A$, and $\partial g /\left.\partial s\right|_{(s, 0)}$ is nearly $-V$ for $s \in B$. It follows that $g(s, 0)$ is close to $g(\alpha, 0)$ for all $s \in[\alpha, \beta]$. That is, the portion of the central curve from $\alpha$ to $\beta$ is nearly stationary like our example. From (6) and (9) we see that $\beta-\alpha$ is nearly $\pi$. Hence all the above reasoning also applies to the interval $[\beta, \alpha+2 \pi]$ in place of $[\alpha, \beta]$. 
Summing up, we have shown that if $G$ is an inversion, and $h$ is sufficiently close to 1 , then at some intermediate time $\tau \in[0,1]$, the immersion $g_{\tau}$ essentially folds $C_{h}$ back and forth, the central curve is nearly stationary and has portions of arbitrarily high curvature "rather densely spread over the curve", and the boundary curves nearly lie on a circle of radius $h$.

14. Stiff cylinders are harder to invert. We can draw a corollary to the analysis of $\S 13$. If we restrict our immersions of $C_{h}$ by insisting that the curvature of the central curve is less than a fixed bound (or equivalently, that the mean curvature is bounded), then we cannot invert $C_{h}$ with $h$ near 1 . Such a bound on the mean curvature would correspond to a stiffness of the material of a physical model for $C_{h}$. Hence stiff cylinders are harder to invert. Stated precisely we have

14.1. TheOREM. For each $B>0, \sup \left\{h \mid C_{h}\right.$ is invertible through isometric immersions whose mean curvatures are bounded by $B\}<1$.

15. Immersing the Möbius strip is strictly easier than embedding it. Here we will draw a corollary to the analysis of $\$ 13$ for the Möbius strip. The results of $\$ 13$ concerning $q_{\tau}$ clearly carry over to any immersion of the Möbius strip $M_{l}=I \times[0, l]$ with $l$ near $\pi / 2$. We will use these results to prove the following theorem.

15.1. THEOREM. There exists an $\varepsilon>0$ such that $M_{l}$ embeds in $\mathbf{R}^{3}$ only if $l>\pi / 2+\varepsilon$.

Proof. Let $k: M_{l} \rightarrow \mathbf{R}^{3}$ be an isometric embedding. We may suppose the plane $P$ described in $\S 13$ (but now related to $k$ ) is the $x y$-plane. Then for $l$ sufficiently close to $\pi / 2$ we have the generating lines nearly horizontal and their centers nearly at one point. Also, $\varphi(t+l / 2)$ nearly makes an angle of $\pi / 2$ with $\varphi(t)$. Hence, the projections of the line segments $E=[\tilde{B}(t), \tilde{T}(t)]$ and $F=[\tilde{B}(t+l / 2), \tilde{T}(t+l / 2)]$ on the $x y$-plane must intersect at a unique point $p$. Let $q \in E$ and $r \in F$ be the points of $E$ and $F$ which lie over $p$. Set $v(t)=$ the vertical distance from $q$ to $r$. Since $k$ is an embedding, we have $v(t) \neq 0$ for all $t \in[0, l / 2]$. It is easy to verify that $v(t)$ is continuous in $t$. Hence $v(0)$ has the same sign as $v(l / 2)$. But clearly $v(0)=-v(l / 2)$, and so we have a contradiction. Hence, for $l$ sufficiently near $\pi / 2, M_{l}$ cannot be isometrically embedded in $\mathbf{R}^{3}$. This proves the theorem. Q.E.D.

Theorem 15.1 suggests the following conjecture.

CONJECTURE. There exists an $\varepsilon>0$ such that $i$ is isometrically isotopic to $r$ only if $h<1-\varepsilon$. 


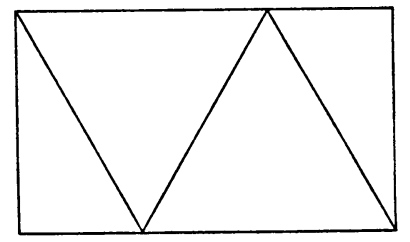

FIGURE 15.1

A paper Möbius strip may be made by folding the strip of paper illustrated in Figure 15.1 along the lines indicated. Using this and the technique of pseudofolds it is easy to see that $M_{l}$ isometrically embeds in $\mathbf{R}^{3}$ if $l>\sqrt{3} . \mathbf{M}$. Sadowsky has given a similar embedding [7], [11]. For isometric but not smooth embeddings of arbitrarily short Möbius strips, see [1, Chapter 3].

CONJECTURE. $M_{l}$ isometrically embeds in $\mathbf{R}^{3}$ only if $l>\sqrt{3}$.

\section{REFERENCES}

1. S. Barr, Experiments in topology, Crowell, New York, 1964.

2. S.-S. Chern and R. K. Lashof, On the total curvature of immersed manifolds, Amer. J. Math. 79 (1957), 306-318. MR 18, 927.

3. S. Eilenberg and D. Montgomery, Fixed point theorems for multi-valued transformations, Amer. J. Math. 68 (1946), 214-222. MR 8, 51.

4. M. L. Gromov and V. A. Rohlin, Imbeddings and immersions in Riemannian geometry, Uspehi Mat. Nauk 25 (1970), no. 5 (155), 3-62 = Russian Math. Surveys 25 (1970), no. 5, 1-57. MR 44 \# 7571.

5. P. Hartman and L. Nirenberg, On spherical image maps whose Jacobians do not change sign, Amer. J. Math. 81 (1959), 901-920. MR 23 \# A4106.

6. D. Laugwitz, Differential and Riemannian geometry, Academic Press, New York, 1965. MR 30 \#2406.

7. M. Sadowsky, Ein elementarer Beweis für die Existenz eines abwickelbaren Möbiusschen Bands und Zurückführung des geometrischen Problems auf ein Variationsproblem, Sitzgber. Preuss. Akad. Wiss. 22 (1930), 412-415.

8. E. H. Spanier, Algebraic topology, McGraw-Hill, New York, 1966. MR 35 \# 1007.

9. J. J. Stoker, Jr., Differential geometry, Interscience, New York, 1969. MR 39 \#2072.

10. H. Whitney, On regular closed curves in the plane, Compositio Math. 4 (1937), 276-284.

11. W. Wunderlich, Uber ein abwickelbares Möbiusband, Monatsh. Math. 66 (1962), 276-289. MR 26 \#680.

DePARTMENT OF Mathematics, INDIANA UNIVERSITY, BLOOMINGTON, INDIANa 47401

COMPUTER Based Education Laboratory, University of IllinoIs, URbana, IllinoIs 61801 